\title{
On the current-voltage relationship in fluid theory
}

\author{
P. Janhunen \\ Finnish Meteorological Institute, Geophysical Research, PO Box 503, FIN 00101, Helsinki, Finland
}

Received: 11 January 1998/Revised: 2 June 1998 / Accepted: 11 June 1998

\begin{abstract}
The kinetic theory of precipitating electrons with Maxwellian source plasma yields the well-known current-voltage relationship (CV-relationship; Knight formula), which can in most cases be accurately approximated by a reduced linear formula. Our question is whether it is possible to obtain this CV-relationship from fluid theory, and if so, to what extent it is physically equivalent with the more accurate kinetic counterpart. An answer to this question is necessary before trying to understand how one could combine time-dependent and transient phenomena such as Alfvénic waves with a slowly evolving background described by the CV-relationship. We first compute the fluid quantity profiles (density, pressure etc.) along a flux tube based on kinetic theory solution. A parallel potential drop accumulates plasma (and pressure) below it, which explains why the current is linearly proportional to the potential drop in the kinetic theory even though the velocity of the accelerated particles is only proportional to the square root of the accelerating voltage. Electron fluid theory reveals that the kinetic theory results can be reproduced, except for different numerical constants, if and only if the polytropic index $\gamma$ is equal to three, corresponding to one-dimensional motion. The convective derivative term $\mathbf{v} \cdot \nabla \mathbf{v}$ provides the equivalent of the "mirror force" and is therefore important to include in a fluid theory trying to describe a CV-relationship. In one-fluid equations the parallel electric field, at least in its functional form, emerges selfconsistently. We find that the electron density enhancement below the potential drop disappears because the magnetospheric ions would be unable to neutralize it, and a square root CV-relationship results, in disagreement with kinetic theory and observations. Also, the potential drop concentrates just above the ionosphere, which is at odds with observations as well. To resolve this puzzle, we show that considering outflowing ionospheric ions restores the possibility of having the acceleration region well above the ionosphere, and thus the electron kinetic (and fluid, if $\gamma=3$ ) theory results are reproduced in a self-consistent manner. Thus the inclu-
\end{abstract}

sion of ionospheric ions is crucial for a feasible CVrelationship in fluid theory. Constructing a quantitative fluid model (possibly one-fluid) which reproduces this property would be an interesting task for a future study.

Key words. Ionosphere (ionosphere-magnetosphere interactions; particle precipitation) - Magnetospheric physics (magnetosphere-ionosphere interactions)

\section{Introduction}

By studying the adiabatic motion of electrons (ions can be similarly treated but their contribution is small) along auroral flux tubes, it can be shown that the current density carried by the precipitating electrons at the ionospheric level is, to a good approximation, given by

$j^{(i)}=K\left(\frac{T_{e}}{e}+V\right)$

where

$K=\frac{n e^{2}}{\sqrt{2 \pi m_{e} T_{e}}}$

is the field-aligned conductance, $T_{e}$ is the electron temperature in energy units, and $V$ is the field-aligned potential drop (corresponding to upward pointing electric field) (Knight, 1973; Lundin and Sandahl, 1978; Fridman and Lemaire, 1980; Janhunen and Olsson, 1998).

Equation (1) is derived by assuming an isotropic Maxwellian source plasma distribution at the equatorial plane and assuming that all electrons which mirror below the ionospheric altitude are captured by the ionosphere, and therefore carry a net negative current into the ionosphere (i.e., net upward current). For this reason, Eq. (1) gives a nonzero current even if the 
potential drop $V$ is zero. We must also assume that some process in the magnetosphere constantly refills the loss cone during every bounce period, so that the distribution function remains isotropic. If the loss cone filling is incomplete, Eq. (1) must be multiplied by the loss cone filling rate (a dimensionless number between 0 and 1 , with unity corresponding to complete loss cone filling) (Janhunen, 1996; Olsson, 1997).

Our subject is to study to what extent the physics behind Eqs. (1) and (2) can be captured in some type of fluid theory. In other words, is it possible to derive these equations from some fluid theory, perhaps in some approximate sense. Just by looking at the symbols appearing in Eq. (2), two things are clear:

1. The equation contains $m_{e}$, thus electron inertia must be included in any fluid theory that tries to reproduce Eqs. (1) and (2).

2. The equation contains $\pi$. There is no "easy" way this natural constant can emerge from a fluid theory, thus it is likely that we can, at best, produce only some approximation to Eq. (2), perhaps an equation that has the same dependence on the physical quantities but a different numerical constant in front of it.

These considerations mean that it is necessary, but maybe not sufficient, to include electron inertia. Likewise, it is not clear how well the numerical factors can be reproduced. Thus an explicit computation is necessary to answer the questions. In order to compare the kinetic and fluid theories in detail, it is necessary to compute the fluid quantity profiles (i.e., profiles along the flux tube) from the exact kinetic theory solution, using the definitions of the fluid quantities as moments of the distribution function. This computation is somewhat tedious and has not been done before. This is the main reason why formulas appearing in the following section are sometimes lengthy.

\section{Kinetic theory}

Consider a general gyrotropic source plasma distribution function $f$ in the equatorial plane, which is symmetric in the field-aligned direction. We use the following normalization:

$$
\begin{aligned}
N_{e} & =\int_{\mathrm{d}^{3} \mathbf{v} f\left(\frac{1}{2} m v_{\|}^{2}, \frac{1}{2} m \mathbf{v}_{\perp}^{2}\right)} \\
& =\int_{0}^{2 \pi} \mathrm{d} \varphi_{v} \int_{-\infty}^{\infty} \mathrm{d} v_{\|} \int_{0}^{\infty} \mathrm{d} v_{\perp} v_{\perp} f\left(\frac{1}{2} m v_{\|}^{2}, \frac{1}{2} m v_{\perp}^{2}\right) \\
& =2 \pi \int_{0}^{\infty} \int_{0}^{\infty} f\left(W_{\|}, W_{\perp}\right) \sqrt{\frac{2}{m^{3} W_{\|}}} \mathrm{d} W_{\perp} \mathrm{d} W_{\|}
\end{aligned}
$$

(we made the obvious changes of variables $W_{\|}=\frac{1}{2} m v_{\|}^{2}$, $W_{\perp}=\frac{1}{2} m v_{\perp}^{2}$, and used symmetry for negative $\left.v_{\|}\right)$. Here $N_{e}$ is the source plasma density. Our $f$ is a function of the parallel and perpendicular kinetic energies $W_{\|}$and $W_{\perp}$.
The particles will be electrons in practice, but this is not essential for the formulas of this section. In this section we will compute the current density as $j=e n v$, i.e., assuming that the particles have positive charges. Thus, a precipitating current will become a positive quantity, which is convenient. When we apply the formulas in later sections we will change the sign for electrons.

For simplicity, we limit ourselves to the Maxwellian source plasma distribution function, which is given by

$f_{M}\left(W_{\|}, W_{\perp}\right)=N_{e}\left(\frac{m}{2 \pi T}\right)^{3 / 2} \exp \left(-\frac{W_{\|}+W_{\perp}}{T}\right)$

and obeys normalization Eq. (3). Here $T$ is the source plasma temperature in energy units. Other distribution functions have been considered before (Pierrard, 1996; Janhunen and Olsson, 1998).

Consider particle motion in one of the hemispheres and let it be the Northern hemisphere. We denote positive parallel velocities as being towards the ionosphere. Half of the particles belonging to the source plasma have a positive velocity. Some of these particles (those with small enough pitch angle) will reach the ionosphere, while others will mirror above the ionosphere. We assume that the ionosphere is a plane at a definite altitude which absorbs all entering particles, and that it emits no particles. We also assume that there exists a potential drop along the magnetic field. The particle kinetic energy is therefore incremented by $\mathrm{eV}$ with $V \geq 0$ if it moves from the equatorial plane to the ionosphere. We denote $u \equiv \mathrm{eV} / T$ ( $u$ is thus the dimensionless potential drop). We choose the normalization of the potential so that $V$ (and $u$ ) vanishes at the equatorial plane. The model is essentially similar of that employed in earlier works (Fridman and Lemaire, 1980; Janhunen and Olsson, 1998) and is shown schematically in Fig. (1).

If $B_{m}$ denotes the equatorial plane magnetic field and $B_{i}$ the ionospheric magnetic field, then $b=B / B_{m}$ is the magnetic field ratio at a general point between the ionosphere and the equatorial plane, and $b_{0}=B_{i} / B_{m}$ is the corresponding quantity at the ionosphere. Clearly

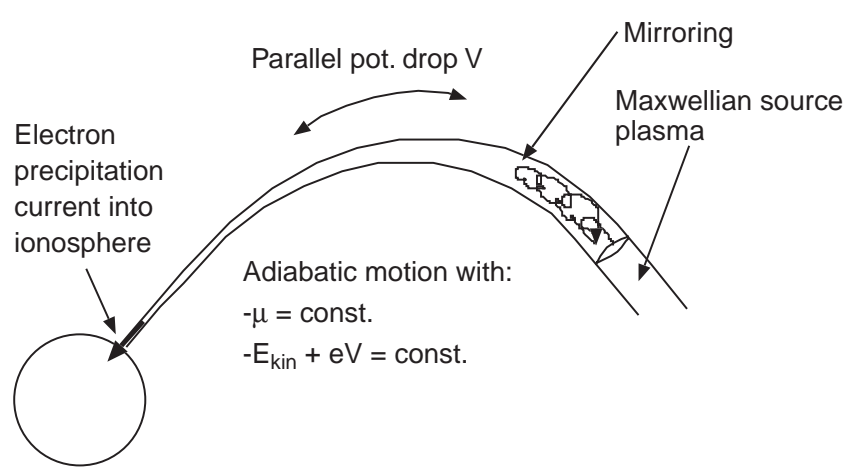

Fig. 1. Schematic description of the kinetic electron model. A Maxwellian source plasma is assumed to be maintained at a high magnetospheric region. Downward of that the first adiabatic invariant $\mu=W_{\perp} / B$ and particle energy are conserved. Particles that hit the ionosphere are assumed to be lost and they form the precipitation current 
we must have $1 \leq b \leq b_{0}$, in analogy with the definition $u_{0}=e V_{0} / T$, where $V_{0}$ is the ionospheric potential.

The downgoing particle flux depends only on source plasma properties, not on the presence of the ionosphere in any way. The upgoing mirrored flux is similar to the downgoing flux, except for a different sign of the parallel velocity (the sign change is equivalent to a time reversal) and the presence of the loss cone. We can compute both upgoing and downgoing fluxes at once by first computing a downgoing flux $\Phi$ while assuming a loss cone in the source plasma (the width of the loss cone is parameterized by $b_{0}$ ). The upgoing mirrored flux is obtained from $\Phi$ by a sign change, because the loss cone was already implemented in the source plasma. The downgoing primary flux is obtained from $\Phi$ by taking the limit $b_{0} \rightarrow \infty$, that is, by removing the loss cone. Equations (19) and (20) later are derived using this methodology.

To provide a hint on how to compute the energydependent particle flux $\Phi$ we first recall how to compute the current density (Janhunen and Olsson, 1998; Fridman and Lemaire, 1980):

$j=2 \pi \int_{0}^{\infty} \int_{0}^{W_{\perp}^{\max }} f\left(W_{\|}, W_{\perp}\right) \sqrt{\frac{2}{m^{3} W_{\|}}}\left(\frac{1}{2} b e v_{\|}\right) \mathrm{d} W_{\perp} \mathrm{d} W_{\|}$,

where the finiteness of

$W_{\perp}^{\max }=\frac{W_{\|}+e V}{b-1}$

removes mirrored particles from the population. This expression for $W_{\perp}^{\max }$ is valid only if the condition

$\frac{V\left(s^{\prime}\right)}{V(s)} \geq \frac{b\left(s^{\prime}\right)-1}{b(s)-1} \quad$ whenever $\quad s^{\prime} \leq s \quad\left[b\left(s^{\prime}\right) \leq b(s)\right]$

( $s$ is the field-aligned coordinate, being zero at the source plasma and growing towards the ionosphere) is valid along the field line. We call this condition the FridmanLemaire condition (FL-condition) (Fridman and Lemaire, 1980). For example, if $V$ is a linear function of the magnetic field, $V(b)=A(b-1)$ where $A$ is a constant, the FL-condition is satisfied (in fact, marginally satisfied). If the FL-condition is not satisfied, some particles which would precipitate according to Eqs. (5) and (6) will actually mirror. For example, if the parallel electric field is zero in the vicinity of the equatorial plane, particles with very small parallel energy and perpendicular energy smaller than the potential drop would reach the ionospheric altitude according to Eqs. (5) and (6), but will in reality mirror close to the source region.

If the potential drop is concentrated in the acceleration region at about $1 R_{E}$ altitude, the FL-condition is not necessarily in force above the acceleration region and exact calculations would then become complicated and dependent on the details of the potential distribution, i.e., on exactly how and where FL-condition is violated (Whipple, 1978). To estimate how much our results could change if the FL-condition is violated, assume that the potential drop is totally confined below a certain altitude, $2 R_{E}$, say, but that below that altitude, the potential drop distribution is such that the FLcondition (7) is in force there. Then the particle motion above the potential drop is unaffected by the potential drop and we could as well assume that the region above that altitude is, in fact, source plasma. That is, we can use our formulas derived later, just changing (decreasing) the values of $b$ and $b_{0}$. For source plasma at $2 R_{E}$ altitude, $b_{0}$ becomes about 30 , which is still so large that our $1 / b_{0}$ expansions will still be valid. That is, our results will be practically unaffected by such a violation of the FL-condition. It is physically evident that the case where the FL-condition is violated in such a way that the potential drop is totally confined below a certain altitude is the worst case, thus we have shown that our results are not, in practice, affected by possible violations of the FL-condition.

Equation (5) is the same as the normalization integral (Eq. 3) except that the factor $\left(e v_{\|}(1 / 2) b\right)$ has been inserted and the integration domain has been reduced to include only those particles that have small enough pitch angles to penetrate the ionosphere (parameter $W_{\perp}^{\max }$ ). The parallel velocity $v_{\|}$is given by $v_{\|}=\sqrt{2 W_{\|} / m}$. The explanations of different factors in $\left(e v_{\|}(1 / 2) b\right)$ are as follows: $e v_{\|}$gives us the current density, $(1 / 2)$ excludes those particles that approach the other hemisphere, and $b=B / B_{m}$ takes into account the scaling of the flux tube so that particles are conserved. Notice that the integral is performed in the equatorial plane. Therefore it is the equatorial plane parallel velocity $v_{\|}$which appears in the formula, not the ionospheric parallel velocity. The flux (current) is still the same as that hitting the ionosphere because we have determined the loss cone in the equatorial plane analytically and limited the domain of integration accordingly.

\subsection{General particle flux}

We are now ready to write an expression for the energydependent particle flux $\Phi$ with general source plasma distribution. We just generalize Eq. (5) by inserting two Dirac $\delta$-functions:

$$
\begin{aligned}
\Phi\left(E_{\|}, E_{\perp}\right)= & \int_{0}^{\infty} \mathrm{d} W_{\|} \int_{0}^{W_{\perp}^{\max }} \mathrm{d} W_{\perp} f\left(W_{\|}, W_{\perp}\right) \frac{C}{\sqrt{W_{\|}}} \\
& \times\left(\frac{1}{2} b \sqrt{\frac{2 W_{\|}}{m}}\right) \delta\left(W_{\|}^{\prime}-E_{\|}\right) \delta\left(W_{\perp}^{\prime}-E_{\perp}\right)
\end{aligned}
$$

where $C \equiv 2 \pi \sqrt{2} m^{-3 / 2}$ and $W_{\|}^{\prime}$ and $W_{\perp}^{\prime}$ are the parallel and perpendicular kinetic energies of a particle accelerated through the potential drop $V$ and penetrating into the convergent magnetic field $b . W_{\|}$and $W_{\perp}$ are the source plasma particle energies and $E_{\|}$and $E_{\perp}$ are the energies observed by a detector at magnetic field strength $b$. Assuming conservation of energy and the first adiabatic invariant $\mu \equiv W_{\perp} / B$ we obtain 


$$
\begin{aligned}
W_{\perp}^{\prime} & =b W_{\perp} \\
W_{\|}^{\prime} & =W_{\|}+u T-(b-1) W_{\perp} .
\end{aligned}
$$

Doing the $W_{\perp}$ integration by using one of the $\delta$ functions we obtain

$$
\begin{aligned}
& \Phi\left(E_{\|}, E_{\perp}\right)= \frac{1}{2} \\
& C \sqrt{\frac{2}{m}} \int_{0}^{\infty} \mathrm{d} W_{\|} f\left(W_{\|}, \frac{1}{b} E_{\perp}\right) \\
& \times \delta\left(W_{\|}+(1-b) \frac{1}{b} E_{\perp}+u T-E_{\|}\right) \\
& \times \theta\left(W_{\perp}^{\max }-\frac{1}{b} E_{\perp}\right)
\end{aligned}
$$

where $\theta(x)$ is the Heaviside theta function (unit step function, defined by $\theta(x)=1$ for $x>0$ and $\theta(x)=0$ for $x<0)$.

The $\theta$-function requires $W_{\|} \geq(1-1 / b) E_{\perp}-u T$. The remaining $\delta$-function, on the other hand, dictates $W_{\|}=(1-1 / b) E_{\perp}-u T+E_{\|}$. Thus we see that, since $E_{\|} \geq 0$, the $\theta$-function is always unity and thus can be dropped. However, we get a new $\theta$-function from the requirement that the singularity of the $\delta$-function is in the $W_{\|}$integration domain, i.e., that $W_{\|} \geq 0$. Finally we obtain

$$
\begin{aligned}
\Phi\left(E_{\|}, E_{\perp}\right)= & \frac{2 \pi}{m^{2}} f\left(\left(1-\frac{1}{b}\right) E_{\perp}-u T+E_{\|}, \frac{1}{b} E_{\perp}\right) \\
& \times \theta\left(\left(1-\frac{1}{b}\right) E_{\perp}-u T+E_{\|}\right) .
\end{aligned}
$$

\subsection{Particle flux for a Maxwellian with a loss cone}

We now compute the flux (11) with $f$ being a Maxwellian with a loss cone. The loss cone is introduced in the equatorial plane particle distribution function $f$ by inserting the factor $\theta\left(\left(b_{0}-1\right) W_{\perp}-W_{\|}-e V_{0}\right)$. Thus

$$
f\left(W_{\|}, W_{\perp}\right)=f_{M}\left(W_{\|}, W_{\perp}\right) \theta\left(\left(b_{0}-1\right) W_{\perp}-W_{\|}-e V_{0}\right) .
$$

Substituting Eq. (12) into Eq. (11) we obtain

$$
\begin{aligned}
\Phi\left(E_{\|}, E_{\perp}\right)= & \frac{2 \pi}{m^{2}} f_{M}\left(E_{\|}+\left(1-\frac{1}{b}\right) E_{\perp}-e V, \frac{1}{b} E_{\perp}\right) \\
& \times \theta\left(\left(b_{0} / b-1\right) E_{\perp}-E_{\|}-e\left(V_{0}-V\right)\right) \\
& \times \theta\left((1-1 / b) E_{\perp}-e V+E_{\|}\right) .
\end{aligned}
$$

We can now compute various fluid quantities by multiplying $\Phi\left(E_{\|}, E_{\perp}\right)$ by suitable expressions and integrating over the energies. Let us introduce the general notation for any function $g$ :

$\langle g\rangle \equiv \int_{0}^{\infty} \int_{0}^{\infty} \mathrm{d} E_{\|} \mathrm{d} E_{\perp} g \Phi\left(E_{\|}, E_{\perp}\right)$.

For example, to compute the current density associated with the particle flux we just use $e$ : $j=\langle e\rangle \equiv e \int_{0}^{\infty} \int_{0}^{\infty} \mathrm{d} E_{\|} \mathrm{d} E_{\perp} \Phi\left(E_{\|}, E_{\perp}\right)$.

Inspection of the $\theta$-functions in Eq. (13) gives us the following rule for the computation of a quantity $\langle g\rangle$.

$$
\begin{aligned}
\langle g\rangle= & \left(\int_{\frac{b}{b_{0}-1} e V_{0}}^{\frac{e V}{1-1 / b}} \mathrm{~d} E_{\perp} \int_{e V-\left(1-\frac{1}{b}\right) E_{\perp}}^{\left(\frac{b_{0}}{b}-1\right) E_{\perp}-e\left(V_{0}-V\right)} \mathrm{d} E_{\|}\right. \\
& \left.+\int_{\frac{e V}{1-\frac{1}{b}}}^{\infty} \mathrm{d} E_{\perp} \int_{0}^{\left(\frac{b_{0}}{b}-1\right) E_{\perp}-e\left(V_{0}-V\right)} \mathrm{d} E_{\|}\right) \\
& \times f_{M}\left(E_{\|}+\left(1-\frac{1}{b}\right) E_{\perp}-e V, \frac{1}{b} E_{\perp}\right) g .
\end{aligned}
$$

The integration domain is visualized in Fig. 2. A similar energy-space figure was previously used by Whipple (1978) while other authors have used velocity space figures (e.g., Chiu and Schulz, 1978).

\subsection{Fluid quantity profiles}

In this section we apply Eq. (16) to compute the current density, parallel velocity and density.

The current density is

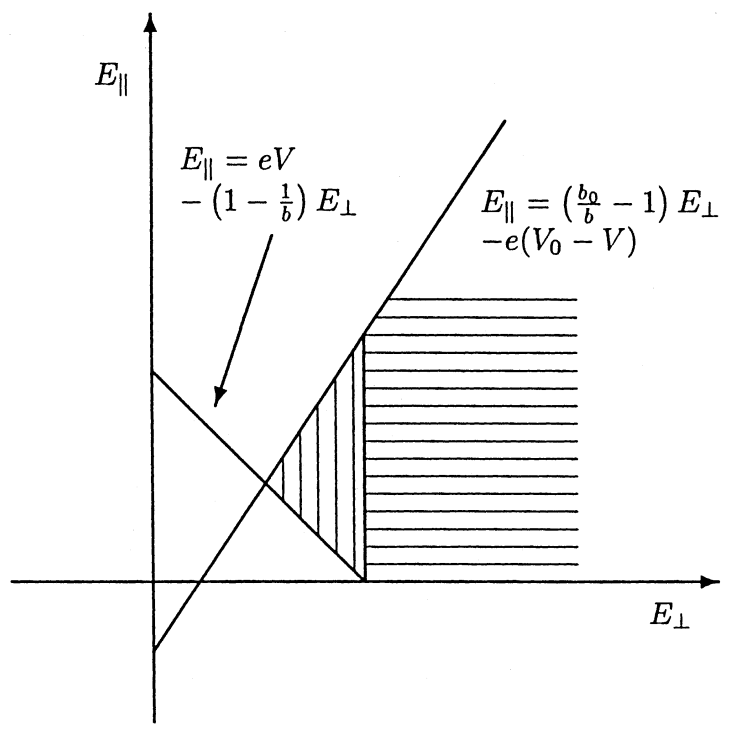

Fig. 2. The integration domain in Eq. (16). The vertical hatching corresponds to the first term and the horizontal hatching the second term. The domain is limited by two lines, the equations of which are shown, together with the $E_{\perp}$ axis. As long as the FL-condition (7) remains valid, the topology of the domain does not change 


$$
\begin{aligned}
j=\langle e\rangle=e N_{e} \sqrt{\frac{T}{2 \pi m}}[ & -(b-1) \mathrm{e}^{-\left(\frac{u}{b-1}\right)} \\
& \left.+b\left(1-\frac{1}{b_{0}}\right) \mathrm{e}^{-\left(\frac{u_{0}}{b_{0}-1}\right)}\right]
\end{aligned}
$$

For the particle density we obtain

$$
\begin{aligned}
n= & \left\langle\frac{1}{v_{\|}}\right\rangle=\left\langle\sqrt{\frac{m}{2 E_{\|}}}\right\rangle \\
= & \frac{1}{2} N_{e}\left\{\sqrt{1-\frac{b}{b_{0}}} \mathrm{e}^{\frac{b_{0} u-b u_{0}}{b_{0}-b}} \operatorname{erfc}\left(\sqrt{\frac{\left(b_{0}-1\right) u-(b-1) u_{0}}{\left(1-1 / b_{0}\right)\left(b_{0}-b\right)}}\right)\right. \\
& \left.+\sqrt{b-1} \mathrm{e}^{-\left(\frac{u}{b-1}\right)} \operatorname{erfi}\left(\sqrt{\frac{u}{b-1}-\frac{u_{0}}{b_{0}-1}}\right)\right\}
\end{aligned}
$$

Here erf is the error function (Abramowitz and Stegun, $1972), \operatorname{erf}(z) \equiv(2 / \sqrt{\pi}) \int_{0}^{z} d t e^{-t^{2}}$, erfc is the complementary error function, $\operatorname{erfc}(z) \equiv 1-\operatorname{erf}(z)$, and erfi is the error function at imaginary argument, $\operatorname{erfi}(z) \equiv$ $-i \operatorname{erf}(i z)$. The arguments of the square roots will remain non-negative because of the FL-condition (7). Notice that $\operatorname{erfc}(0)=1$ and $\operatorname{erfi}(0)=0$.

The currents and densities associated with down- and upgoing populations are obtained from (see discussion before Eq. 5)

$j^{\text {down }}=\lim _{b_{0} \rightarrow \infty} j, \quad n^{\text {down }}=\lim _{b_{0} \rightarrow \infty} n$

$j^{\text {up }}=-j, \quad n^{\text {up }}=n$.

Finally, the total quantities are computed as

$j^{\text {tot }}=j^{\text {down }}+j^{\text {up }}, \quad n^{\text {tot }}=n^{\text {down }}+n^{\text {up }}$.

The upgoing quantities $j^{\text {up }}, n^{\text {up }}$ are identical to Eqs. (17) and (18) except for the sign change. For the downgoing quantities we obtain, after taking the limits,

$$
\begin{aligned}
& j^{\text {down }}=e N_{e} \sqrt{\frac{T}{2 \pi m}}\left[b-(b-1) \mathrm{e}^{-\left(\frac{u}{b-1}\right)}\right] \\
& n^{\text {down }}=\frac{N_{e}}{2}\left\{\mathrm{e}^{u} \operatorname{erfc}(\sqrt{u})+\sqrt{b-1} \mathrm{e}^{-\left(\frac{u}{b-1}\right)} \operatorname{erfi}\left(\sqrt{\frac{u}{b-1}}\right)\right\} .
\end{aligned}
$$

The total particle density is thus the sum of Eqs. (18) and (23), and the total current density is the difference of Eqs. (22) and (17). For the total current density we get

$j^{\text {tot }}=e N_{e} \sqrt{\frac{T}{2 \pi m}}\left(\frac{b}{b_{0}}\right)\left[b_{0}-\left(b_{0}-1\right) \mathrm{e}^{-\left(\frac{u_{0}}{b_{0}-1}\right)}\right]$,

from which we see that $j^{\text {tot }}$ depends on $b$ linearly, as it should because of current continuity.

To obtain the total pressure $P^{\text {tot }}$ (both upgoing and downgoing contributions included) we proceed as follows. The scalar pressure $P$ is equal to $(1 / 3) \operatorname{tr} \mathscr{P}$, where $\mathscr{P}$ is the pressure tensor:

$$
\begin{aligned}
P & =\frac{m}{3} \int \mathrm{d} \mathbf{v}(\mathbf{v}-\mathbf{u})^{2} f \\
& =\frac{2}{3} \int \mathrm{d} \mathbf{v}\left(W_{\|}+W_{\perp}\right) f-\frac{1}{3} m n u^{2}
\end{aligned}
$$

where $\mathbf{u}$ is the average velocity $\left(\mathbf{u}=(1 / n) \int \mathrm{d} \mathbf{v v} f\right.$, $\left.n=\int \mathrm{d} \mathbf{v} f\right)$. The integral can be expressed as

$$
\begin{aligned}
& \int \mathrm{d} \mathbf{v}\left(W_{\|}+W_{\perp}\right) f \\
& =\pi \int_{0}^{\infty} \int_{0}^{\infty} \mathrm{d} W_{\|} \mathrm{d} W \perp \sqrt{\frac{2}{m^{3} W_{\|}}}\left(f^{\text {down }}+f^{\text {up }}\right)\left(W_{\|}+W_{\perp}\right) \\
& =\sqrt{\frac{m}{2}} \int_{0}^{\infty} \int_{0}^{\infty} \mathrm{d} E_{\|} \mathrm{d} E_{\perp} \frac{E_{\|}+E_{\perp}}{\sqrt{E_{\|}}}\left(\Phi\left(E_{\|}, E_{\perp}\right)^{\text {up }} .\right. \\
& \left.+\Phi\left(E_{\|}, E_{\perp}\right)^{\text {down }}\right),
\end{aligned}
$$

where we changed the name of the integration variable from $W$ to $E$ and used the fact that $f=\left(m^{2} /(2 \pi)\right) \Phi$ (see e.g., Eq 11; this is the connection between the distribution function $f$ and the particle flux $\Phi$ observed by a detector at the same place). Denoting $F \equiv$ $\left\langle\sqrt{E_{\|}}+E_{\|} / \sqrt{E_{\perp}}\right\rangle$ we have

$P^{\mathrm{tot}}=\frac{1}{3} \sqrt{2 m}\left(F+\lim _{b_{0} \rightarrow \infty} F\right)-\frac{1}{3} m \frac{\left(j^{\mathrm{tot}}\right)^{2}}{\mathrm{e}^{2} n^{\mathrm{tot}}}$.

because the mean velocity $u=j^{\text {tot }} / n^{\text {tot }}$.

For $F$ we obtain

$$
\begin{aligned}
& F=\frac{N_{e} T}{2 \sqrt{2 m}}\left\{-2 b \mathrm{e}^{-\left(\frac{u}{b-1}\right)} \sqrt{\frac{(2 b-1) u}{\pi(b-1)}}\right. \\
& +\frac{2 b}{\sqrt{\pi}} \mathrm{e}^{-\left(\frac{u_{0}}{b_{0}-1}\right)} \\
& \times\left(\sqrt{u+\frac{b u_{0}}{b_{0}-1}}-\sqrt{u-\frac{(b-1) u_{0}}{b_{0}-1}}\right) \\
& +b^{3 / 2} \mathrm{e}^{u / b}\left[\operatorname{erf}\left(\sqrt{\frac{(2 b-1) u}{(b-1) b}}\right)\right. \\
& \left.-\operatorname{erf}\left(\sqrt{\frac{\left(b_{0}-1\right) u+b u_{0}}{b\left(b_{0}-1\right)}}\right)\right] \\
& +\frac{\mathrm{e}^{\frac{b_{0} u-b u_{0}}{b_{0}-b}}\left[3 b_{0}+b\left(2\left(u_{0}-u\right)-3\right)\right]}{\sqrt{b_{0}\left(b_{0}-b\right)}} \\
& \times \operatorname{erf}\left(\sqrt{\frac{\left(b_{0}-1\right) u-(b-1) u_{0}}{\left(1-1 / b_{0}\right)\left(b_{0}-b\right)}}\right) \\
& +\frac{\mathrm{e}^{-\left(\frac{u}{b-1}\right)}\left[1+b^{2}+2 b(u-1)\right]}{\sqrt{b-1}} \\
& \left.\times \operatorname{erfi}\left(\sqrt{\frac{u}{b-1}-\frac{u_{0}}{b_{0}-1}}\right)\right\} \text {. }
\end{aligned}
$$

For the $b_{0} \rightarrow \infty$ limit we obtain 


$$
\begin{aligned}
\lim _{b_{0} \rightarrow \infty} F= & \frac{N_{e} T}{2 \sqrt{2 m}}\left\{-2 b \mathrm{e}^{-\left(\frac{u}{b-1}\right)} \sqrt{\frac{(2 b-1) u}{\pi(b-1)}}\right. \\
& +3 \mathrm{e}^{u} \operatorname{erfc}(\sqrt{u})+b^{3 / 2} \mathrm{e}^{u / b} \\
& \times\left[\operatorname{erf}\left(\sqrt{\frac{(2 b-1) u}{(b-1) b}}\right)-\operatorname{erf}\left(\sqrt{\frac{u}{b}}\right)\right] \\
& +\frac{\mathrm{e}^{-\left(\frac{u}{b-1}\right)}\left[1+b^{2}+2 b(u-1)\right]}{\sqrt{b-1}} \\
& \left.\times \operatorname{erfi}\left(\sqrt{\frac{u}{b-1}}\right)\right\}
\end{aligned}
$$

In Fig 3, 4 and 5 we show the density, pressure and temperature profiles plotted for a specific potential drop model. A potential drop model is fixed by giving its dependence on $b$. Our model is given by

$u(b)=C\left\{-0.5 \times \tanh \left[\frac{\left(b_{0} / b\right)^{1 / 3}-1.5}{0.5}\right]+0.5\right\}$,

with the constant $C$ chosen such that $u\left(b_{0}\right)=u_{0}$, where $u_{0}$ is the desired potential drop that appears in the figure

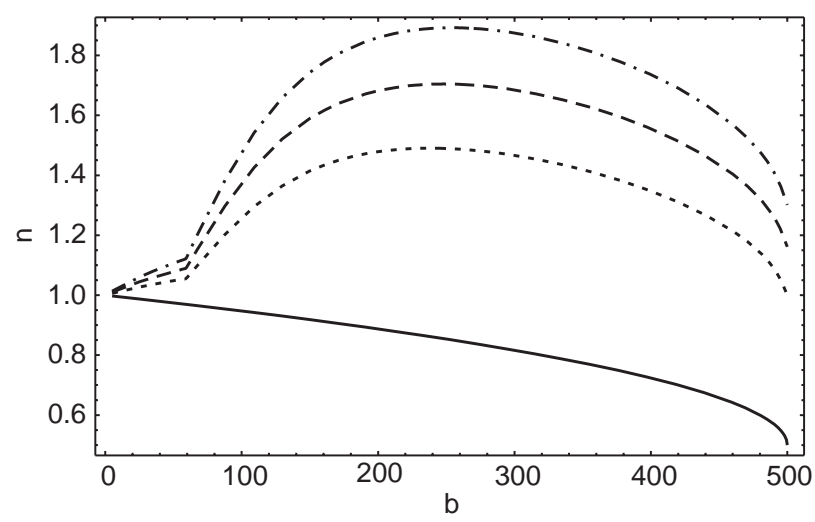

Fig. 3. The profiles of density $n$ for potential drops $0,2,3,4$ corresponding to solid, dotted, dashed, and dot-dashed curves, respectively. The dimensionless potential drops $u$ are normalized to the source plasma thermal energy. The curves were obtained by using Eq. (21)

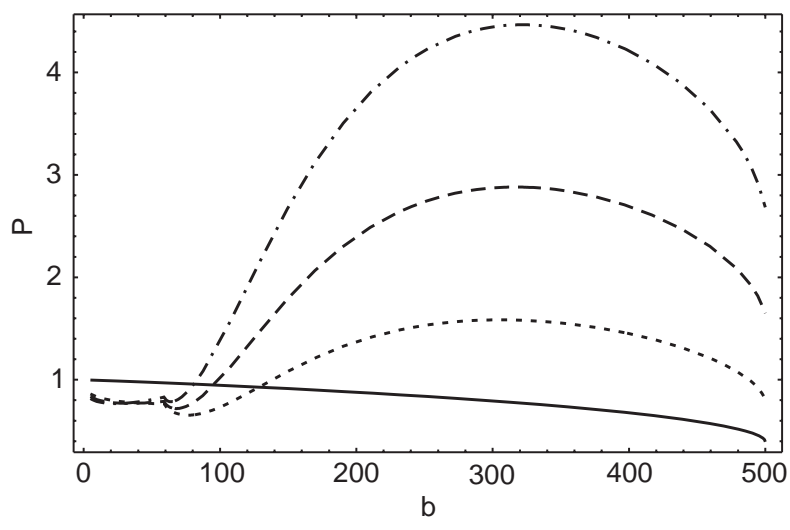

Fig. 4. Same as Fig. 3 but for the pressure (Eq. 27)

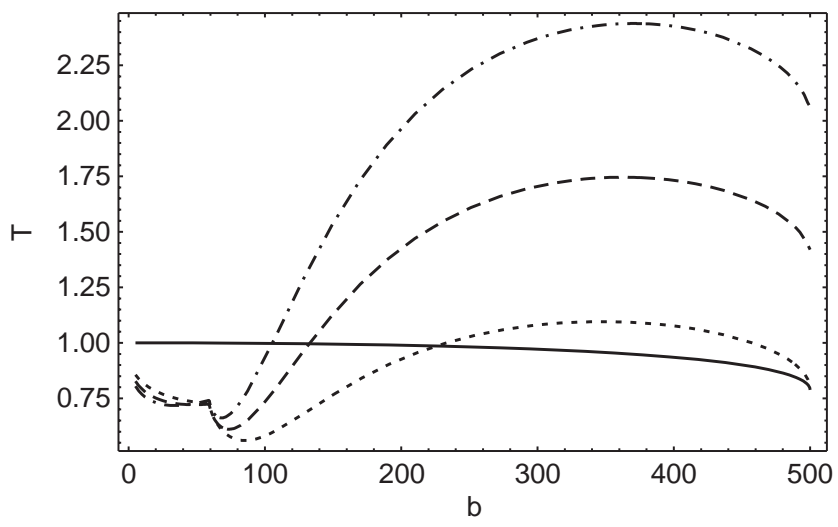

Fig. 5. Same as Fig. 3 but for the temperature

captions. This model sets the acceleration region at roughly $10000 \mathrm{~km}$ altitude from the ionospheric plane, with thickness about $6000 \mathrm{~km}$. The motivation for the power $1 / 3$ is the altitude dependence of the dipole field. In addition, $u(b)$ was not allowed to be smaller than $u_{0} b /\left(b_{0}-1\right)$. This ensures that the FL-condition (7) is in force. The enforcement of this condition has an effect for $b / b_{0}$ smaller than about 70 in our case. The $u(b)$ profile remains continuous but has a kink; consequently some kinks are also seen in the fluid quantity profiles at this abscissa value. Equation (30) for varying $C$ is shown in Fig. 6.

We do not claim that our potential drop model Eq. (30) is necessarily of any physical relevance. It was just used for illustrative purposes to generate Figs. 3-6.

\subsection{The case without a potential drop}

The case without a parallel potential drop provides us with some physical idea of the fluid quantity profiles. Substituting $u=u_{0}=0$ in $n^{\text {tot }}$ gives

$n^{\mathrm{tot}}=\frac{N_{e}}{2}\left(1+\sqrt{1-\frac{b}{b_{0}}}\right) \quad\left(u=u_{0}=0\right)$

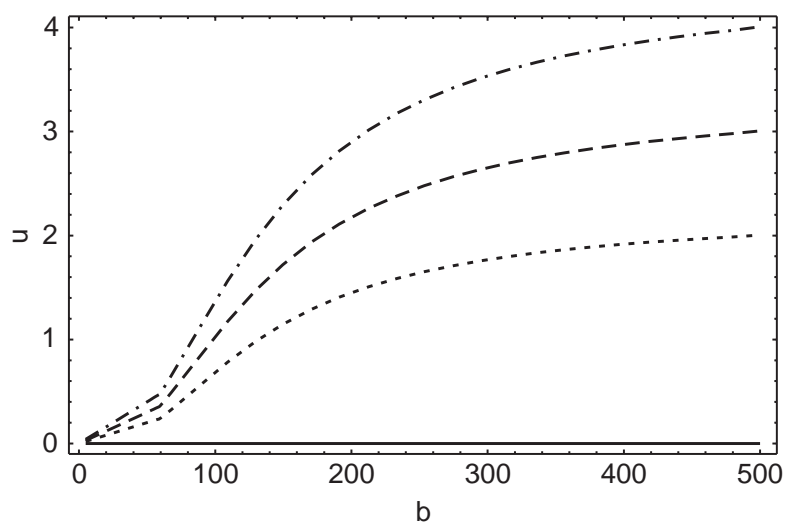

Fig. 6. Same as Fig. 3, but now displaying the potential drop model itself (Eq. 30) 
which gives $N_{e}$ at the source region $(b=0)$ and $N_{e} / 2$ at the ionosphere $\left(b=b_{0}\right)$. In between the profile is monotonic. The ionosphere captures the downgoing population and thus the particle density has only half its original value. Similarly, for the current density we find

$j^{\mathrm{tot}}=\frac{b}{b_{0}} e N_{e} \sqrt{\frac{T}{2 \pi m}} \quad\left(u=u_{0}=0\right)$.

Thus, at the ionosphere we have the thermal current density $e N_{e} \sqrt{T /(2 \pi m)}$ (which is equivalent to the Jeans escape flux, or the escape flux of kinetic molecular effusion, Gombosi, 1994). Above the ionosphere the current density is defined by current continuity, hence the factor $b / b_{0}$.

For the pressure we obtain

$$
\begin{aligned}
& P^{\mathrm{tot}}=N_{e} T {\left[\frac{1}{2}+\frac{1}{2} \sqrt{1-\frac{b}{b_{0}}}\right.} \\
&\left.-\frac{1}{3 \pi} \frac{b}{b_{0}}\left(1-\sqrt{1-\frac{b}{b_{0}}}\right)\right] \\
&\left(u=u_{0}=0\right) .
\end{aligned}
$$

For $b=0$ (source region) we have $P^{\text {tot }}=N_{e} T$ as we should. For the ionosphere $\left(b=b_{0}\right)$ we have $P^{\text {tot }}=N_{e} T(1 / 2-1 /(3 \pi)) \approx 0.394 N_{e} T$. In between the profile is monotonic, as for the density.

The local temperature profile $T_{\text {loc }} \equiv P / n$ is given by

$$
T_{\mathrm{loc}}=T\left[1-\frac{2}{3 \pi}\left(\frac{b}{b_{0}}\right) \frac{1-\sqrt{1-b / b_{0}}}{1+\sqrt{1-b / b_{0}}}\right] \quad\left(u=u_{0}=0\right)
$$

For the source region $T_{\mathrm{loc}}=T$ as it must be, and for the ionosphere we obtain $T_{\mathrm{loc}}=(1-2 /(3 \pi)) T \approx 0.788 T$. Also the temperature profile is monotonic.

\subsection{Ionospheric variables}

Variables at the ionospheric plane can be obtained by taking the limits $u \rightarrow u_{0}, b \rightarrow b_{0}$ of the total expressions, or by substituting $u \rightarrow u_{0}, b \rightarrow b_{0}$ in the downgoing population quantities only, because the upgoing quantities at ionospheric plane are automatically zero. (For the pressure the latter method cannot be used because we do not have a meaningful up/down decomposition.) We obtain

$$
\begin{aligned}
n^{\text {iono }}= & \frac{N_{e}}{2}\left[\mathrm{e}^{u_{0}} \operatorname{erfc}\left(\sqrt{u_{0}}\right)+\sqrt{b_{0}-1} \mathrm{e}^{-\left(\frac{u_{0}}{b_{0}-1}\right)}\right. \\
& \left.\times \operatorname{erfi}\left(\sqrt{\frac{u_{0}}{b_{0}-1}}\right)\right] \\
j^{\text {iono }}= & e N_{e} \sqrt{\frac{T}{2 \pi m}}\left[b_{0}-\left(b_{0}-1\right) \mathrm{e}^{-\left(\frac{u_{0}}{b_{0}-1}\right)}\right]
\end{aligned}
$$

Let us expand these expressions for large $b_{0}$. Usually $b_{0}$ is more than 100 , so this is a good approximation (Janhunen and Olsson, 1998; Fridman and Lemaire, 1980): $n^{\text {iono }}=\frac{N_{e}}{2}\left[\mathrm{e}^{u_{0}} \operatorname{erfc}\left(\sqrt{u_{0}}\right)+2 \sqrt{\frac{u_{0}}{\pi}}-\frac{4}{3} \frac{u_{0}^{3 / 2}}{b_{0} \sqrt{\pi}}+O\left(\frac{1}{b_{0}^{2}}\right)\right]$

$j^{\text {iono }}=e N_{e} \sqrt{\frac{T}{2 \pi m}}\left[1+u_{0}-\frac{u_{0}^{2}}{2 b_{0}}+O\left(\frac{1}{b_{0}^{2}}\right)\right]$.

The ionospheric density $n^{\text {iono }}$ has been plotted as a function of the potential drop and $b_{0} \rightarrow \infty$ in Fig. 7 . The density increases monotonically as $u_{0}$ increases. For $u_{0}>2.20615$ it becomes larger than the source plasma density.

The pressure expression is more complicated and therefore we choose to compute it only to zeroth order in $1 / b_{0}$ :

$$
\begin{aligned}
P^{\text {iono }}= & \frac{N_{e} T}{3}\left\{\frac{2(1+2 \sqrt{2}) u_{0}^{3 / 2}-3 \sqrt{u_{0}}}{3 \sqrt{\pi}}+\frac{3}{2} \mathrm{e}^{u_{0}} \operatorname{erfc}\left(\sqrt{u_{0}}\right)\right. \\
& \left.-\frac{\left(1+u_{0}\right)^{2}}{\pi\left[\mathrm{e}^{u_{0}} \operatorname{erfc}\left(\sqrt{u_{0}}\right)+2 \sqrt{u_{0} / \pi}\right]}+O\left(\frac{1}{b_{0}}\right)\right\} .
\end{aligned}
$$

The plot of $P^{\text {iono }}$ also appears in Fig. 7. Unlike the density, the pressure is not monotonically increasing but has a minimum of 0.114882 at $u_{0} \approx 0.424345$. For $u_{0}>1.37227$ it becomes larger than the value at $u_{0}=0$ (which is $1 / 2-1 /(3 \pi) \approx 0.393897$ ). For $u_{0}>2.32749$ it becomes larger than the source plasma pressure and then increases more steeply than the density.

\subsection{Conclusion}

We have solved the fluid quantity profiles in the single particle (electron) kinetic theory. This is essentially the same model as was used in previous works (Fridman and Lemaire, 1980; Janhunen and Olsson, 1998), but here we have computed the fluid quantities everywhere along the field line, not just the current that leaks into the ionosphere.

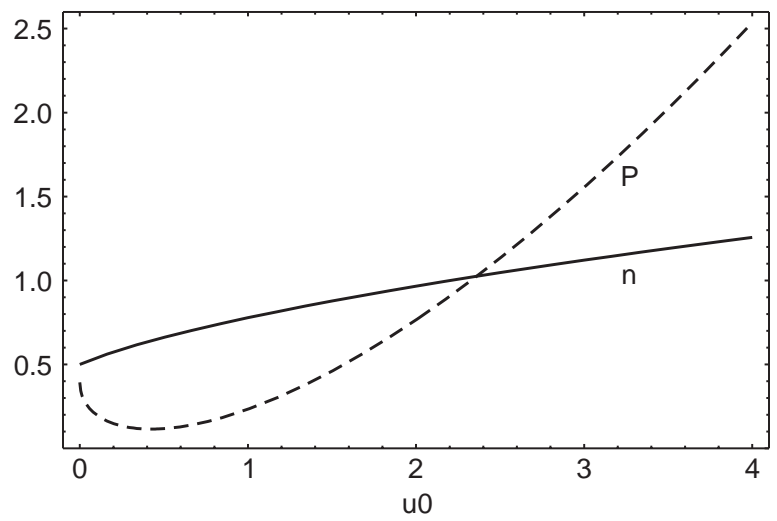

Fig. 7. Ionospheric $n / N_{e}$ and $P /\left(N_{e} T\right)$ as a function of the potential drop $u_{0}=e V_{0} / T$ for $b_{0} \rightarrow \infty$ (Eqs. 37 and 39) 


\section{Electron fluid theory}

We would like to know what is the fluid theory equivalent of Eq. (1), i.e., how to obtain the same effect from either single or two-fluid equations. Because the current is carried essentially only by electrons, it is obvious that it is easiest to start by looking at the electron fluid equations in the two-fluid formalism. We shall drop the subscript $e$ denoting 'electron' in this section.

The adiabatic electron fluid equations, in given electromagnetic field, can be written as

$\nabla \cdot \Pi=0$

$n m \mathbf{v} \cdot \nabla \mathbf{v}=-e n(\mathbf{E}+\mathbf{v} \times \mathbf{B})-\nabla P$

$\mathbf{v} \cdot \nabla\left(\frac{P}{n^{\gamma}}\right)=0$.

Here $\boldsymbol{\Pi} \equiv n \mathbf{v}$ denotes the mass flux vector. We have written the equations in the stationary state. Let us assume that the quantities depend only on the fieldaligned coordinate $s$ and that the vectors $\mathbf{v}$ and $\mathbf{j}$ have only parallel components, denoted by $v_{\|}$and $j_{\|}$, respectively. All the time we are considering a low-beta plasma, i.e., a region where the magnetic energy density is much larger than the thermal pressure. For a low-beta plasma all current is field-aligned current (Janhunen and Koskinen, 1997). We shall also assume that there are no perpendicular flows. This assumption is not necessary for the fluid theory itself, but we shall make it here in order to get a model which can be easily compared with the kinetic theory results. Once the fluid/kinetic "calibration" is understood, we should try to build more comprehensive fluid models where the perpendicular dynamics (possibly 3D) is also included. In this section the parallel electric field is assumed to be known.

We can solve Eqs. (40) and (42) easily. The results are

$\Pi=\Pi_{0}\left(\frac{b}{b_{0}}\right)$

$P=C n^{\gamma}$

where $C$ is a constant given by $C=P_{0} / n_{0}^{\gamma}=T_{0} / n_{0}^{\gamma-1}$. Here $P_{0}, T_{0}$ and $n_{0}$ are the source plasma pressure, temperature and density, respectively, whereas $\Pi_{0}$ and $b_{0}$ are respectively the ionospheric mass flux and normalized magnetic field. Please notice and forgive this small illogicality in our notation.

Let us now establish a simple lemma: If $\mathbf{U}$ and $\mathbf{V}$ are divergenceless vector fields parallel to $\mathbf{B}$ such that $\mathbf{U}=g \mathbf{B}, \mathbf{V}=f \mathbf{B}$ then, because $\nabla \cdot \mathbf{U}=\nabla \cdot \mathbf{V}=0, f$ and $g$ are constant along the field line and

$[\mathbf{U} \cdot \nabla \mathbf{V}]_{\|}=U_{\|} V_{\|} \frac{1}{B} \frac{\mathrm{d} B}{\mathrm{~d} s}=U_{\|} V_{\|} \frac{1}{b} \frac{\mathrm{d} b}{\mathrm{~d} s}$.

With the help of lemma (45) we obtain

$$
\begin{aligned}
{[\mathbf{v} \cdot \nabla \mathbf{v}]_{\|} } & =\left(\frac{\boldsymbol{\Pi}}{n}\right)^{2}\left(\frac{1}{b} \frac{\mathrm{d} b}{\mathrm{~d} s}-\frac{1}{n} \frac{\mathrm{d} n}{\mathrm{~d} s}\right) \\
& =\left(\frac{\boldsymbol{\Pi}}{n}\right)^{2}\left(\frac{1}{b}-\frac{1}{n} \frac{\mathrm{d} n}{\mathrm{~d} b}\right) \frac{\mathrm{d} b}{\mathrm{~d} s}
\end{aligned}
$$

Substituting Eqs. (46), (43) and (44) in (42) and taking the parallel component (which is the only nonzero one due to our assumptions) we obtain

$$
\begin{aligned}
& {\left[C \gamma n^{\gamma-2}-m \Pi_{0}^{2} \frac{1}{n^{3}}\left(\frac{b}{b_{0}}\right)^{2}\right] \frac{\mathrm{d} n}{\mathrm{~d} b}} \\
& +m \Pi_{0}^{2}\left(\frac{b}{b_{0}}\right)^{2} \frac{1}{n^{2}} \frac{1}{b}-e \frac{\mathrm{d} V}{\mathrm{~d} b}=0
\end{aligned}
$$

where the parallel electric field $E_{\|}=-\mathrm{d} V(b) / \mathrm{d} b$. Equation (47) is an exact differential equation (Spiegel, 1968) and it can be integrated directly. Putting in the boundary condition $n(b=1)=n_{0}$ (the source plasma density) we obtain

$C \frac{\gamma}{\gamma-1}\left(n^{\gamma-1}-n_{0}^{\gamma-1}\right)+\frac{m \Pi_{0}^{2}}{2 b_{0}^{2}}\left(\frac{b^{2}}{n^{2}}-\frac{1}{n_{0}^{2}}\right)=e V(b)$.

Equation (48) determines the solution $n(b)$ once the mass flux through the bottom of the flux tube $\Pi_{0}$ is known. Since we do not know $\Pi_{0}$, but are trying to determine it, we must use some additional constraint to fix $\Pi_{0}$. One way to fix it is to require that the ionospheric density $n\left(b_{0}\right)$ has a certain value, let us call it $\alpha n_{0}$, where $\alpha$ is a dimensionless parameter. Doing this, we obtain

$\Pi_{0}=n_{0} \sqrt{\frac{T_{0}}{m}} \sqrt{\frac{\frac{2 \gamma}{\gamma-1}\left(1-\alpha^{\gamma-1}\right)+2 u_{0}}{\frac{1}{\alpha^{2}}-\frac{1}{b_{0}^{2}}}}$

where we have denoted $u_{0} \equiv e V_{0} / T_{0}$ as before. For $u_{0}=0$ (no potential drop) the latter square root expression should be equal to $1 / \sqrt{2 \pi}$ in order to get agreement with the kinetic theory result Eq. (31). For $b_{0} \rightarrow \infty$ (usually a good approximation) and $\gamma=5 / 3$ this happens when $\alpha \approx 0.22471$ or $\alpha \approx 0.947264$. (The smaller one of the roots may not be physically meaningful.)

Thus it is possible to "force-fit" the fluid theory to conform with kinetic theory, at least if we do not have a potential drop. However, being a nonlocal operation (we need to know the source plasma density) this kind of approach is not easy to implement generically as a simulation boundary conditions, for instance.

\subsection{Two-step analytic solution}

It would be good if we had some idea within fluid theory as to what value of $\alpha$ we should use. To facilitate this, let us first notice that if the potential drop resides at a high altitude a similar constraint to the FL-condition (Eq. 7 in kinetic theory), the plasma flow within the region of the potential drop may be neglected because the fluxtube cross-sectional area is much larger than the same quantity at the ionospheric plane, and thus we can drop the term proportional to $\Pi_{0}$ (or, equivalently, we can take the limit $\left.b_{0} \rightarrow \infty\right)$ in Eq. (48). In this region the explicit solution is then

$n(b)=n_{0}\left[1+\left(\frac{\gamma-1}{\gamma}\right) u(b)\right]^{1 /(\gamma-1)}$ 
and at the bottom of the potential drop $u(b)=$ $u\left(b_{0}\right) \equiv u_{0}$. Under these circumstances Eq. (48) describes a force equilibrium between the pressure gradient and the electrostatic force. In the lower region the plasma flow must not be neglected, but there the potential drop is zero. Thus the solution of the problem can be found by solving the problem with $V(b)=0$, using Eq. (50) as source plasma density and 'renormalizing' $b$ and $b_{0}$ to the altitude below the potential drop. Even the renormalized $b_{0}$ will still be fairly large and can still be approximated by $b_{0} \rightarrow \infty$ at the end. This approximation is also a reasonable one to make here because it makes the results independent of the distribution of the potential drop, which was the case in kinetic theory anyway. To summarize, our approach now is to solve the problem in two steps: first considering the stationary compression of plasma by the electric field, then consider the outflow of the compressed plasma into the ionosphere through the bottom of the flux tube without electric field (Fig. 8).

Physically the problem without a potential drop corresponds to flow out of a container through a convergent nozzle (Landau and Lifshitz, 1987; Anderson, 1990), with zero external pressure (because there are no hot electrons in the ionosphere). Then the flow at the "nozzle exit" (the ionosphere) will be "choked", with mass flux per unit area given by Eq. (90.2) of Landau and Lifshitz (1987),

$\Pi_{0}=\hat{n_{0}} \sqrt{\frac{\hat{T}_{0}}{m}} \sqrt{\gamma}\left(\frac{2}{\gamma+1}\right)^{\frac{\gamma+1}{2(\gamma-1)}}$.

The hatted quantities represent the state given by Eq. (50) at $u(b)=u_{0}$. Explicitly,

$\hat{n_{0}}=n_{0}\left[1+\left(\frac{\gamma-1}{\gamma}\right) u_{0}\right]^{1 /(\gamma-1)}$

$\hat{T}_{0}=T_{0}\left[1+\left(\frac{\gamma-1}{\gamma}\right) u_{0}\right]$.

since $T=P / n=C n^{\gamma-1}$.

Combining Eqs. (51), (52) and (53) we obtain for the ionospheric mass flow

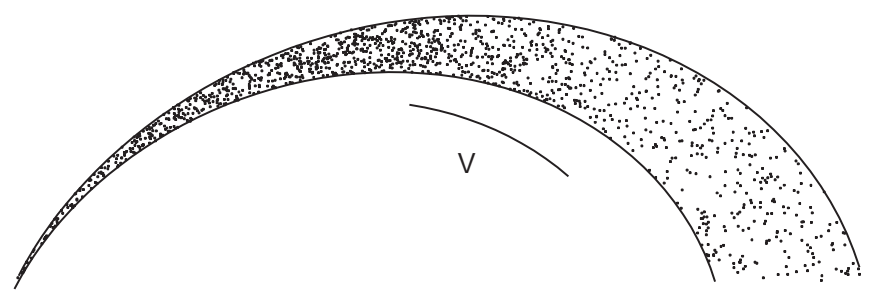

Fig. 8. If the potential drop resides at high enough altitude, it compresses the electron plasma beneath it in such a way that the plasma states above and below the drop are independent of exact geometry of the flux tube. If the ionospheric bottom of the flux tube is narrow enough, the fact that plasma starts escaping into the ionosphere does not appreciably alter the compression by the potential drop $V$

$$
\begin{aligned}
\Pi_{0}= & n_{0} \sqrt{\frac{T_{0}}{m}} \sqrt{\gamma}\left(\frac{2}{\gamma+1}\right)^{\frac{\gamma+1}{2(\gamma-1)}} \\
& {\left[1+\left(\frac{\gamma-1}{\gamma}\right) u_{0}\right]^{\frac{1}{\gamma-1}+\frac{1}{2}} }
\end{aligned}
$$

For $\gamma=5 / 3$ we have

$$
\begin{aligned}
\Pi_{0} & =n_{0} \sqrt{\frac{T_{0}}{m}} \frac{3 \sqrt{15}}{16}\left(1+\frac{2}{5} u_{0}\right)^{2} \\
& \approx 0.73 n_{0} \sqrt{\frac{T_{0}}{m}}\left(1+\frac{2}{5} u_{0}\right)^{2} .
\end{aligned}
$$

For $\gamma=3$ we have

$$
\Pi_{0}=n_{0} \sqrt{\frac{T_{0}}{m}} \frac{\sqrt{3}}{2}\left(1+\frac{2}{3} u_{0}\right) .
$$

We thus notice that $\gamma$ should be equal to 3 in order to obtain a linear current-voltage relationship. Although not directly visible from the plots shown in this paper, we note that if the curves in Fig. 3 are raised to third power, curves rather similar to those in Fig. 4 are obtained, which also suggests that $\gamma=3$ is the closest approximation to the kinetic theory presented here. Of course, we cannot expect the fluid and kinetic theory profiles to be exactly equal.

For $\gamma=5 / 3$ we saw that the relationship became quadratic in $u_{0}$. The various formulas, normalized by $n_{0} \sqrt{T_{0} / m}$, are plotted in Fig. 9 and compared with kinetic theory (Eq. 38). Even for $\gamma=3$ the numerical coefficients are different in kinetic and fluid theories. The thermal current is $\sqrt{3 \pi / 2} \approx 2.17$ times larger in fluid theory. Asymptotically for $u_{0} \gg 1$, the fluid theory current is $\sqrt{2 \pi / 3} \approx 1.45$ times larger.

We thus reach the important conclusion that the electron fluid theory that most resembles the electron kinetic theory, at least as far as the current-voltage relationship is concerned, has $\gamma=3$, corresponding to only one degree of freedom [in general, $\gamma=(2+f) / f$, where $f$ is the number of degrees of freedom]. Apparently the conservation of the first adiabatic invariant effectively removes the two perpendicular degrees of

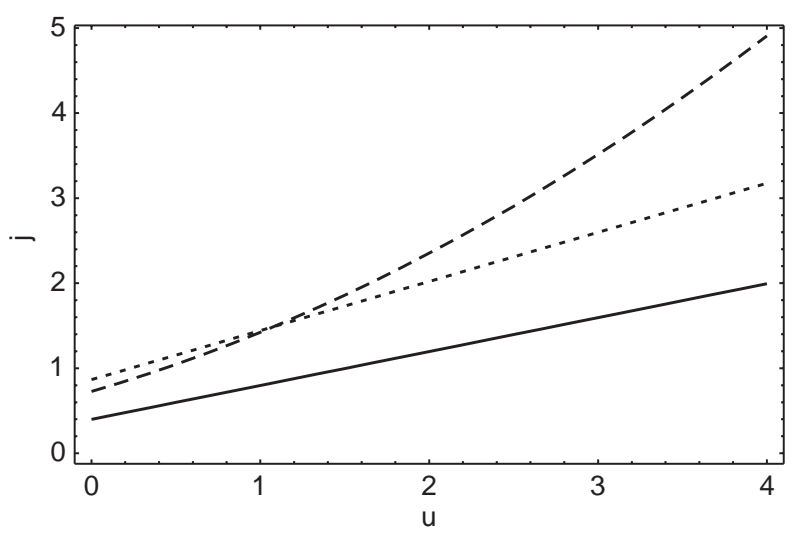

Fig. 9. Comparison of current-voltage relationships in kinetic theory (solid; Eq. 38), fluid theory with $\gamma=3$ (dotted; Eq. 56) and fluid theory with $\gamma=5 / 3$ (dashed; Eq. 55) 
freedom from the electrons, leaving only one degree of freedom. If one tries to use "ordinary" fluid equations with $\gamma=5 / 3$ (3 degrees of freedom), a quadratic current-voltage relationship results, which is in disagreement with both observations (Lundin, 1977; Lyons et al., 1979; Weimer et al., 1987; Lu et al., 1991; Haerendel et al., 1994; Sakanoi et al., 1995; Olsson et al., 1996; Olsson et al., 1998) and kinetic theory.

The exact implications of this finding are not yet quite clear to us. At least in the force-free near-Earth low-beta plasma region (which is the subject of this study), the electrons should have $\gamma=3$ rather than $\gamma=5 / 3$. But how far into the magnetosphere should this result be generalized is not yet so clear. It is also an open question whether or not the ions should also have $\gamma=3$.

An essential feature of both the kinetic theory and the electron-fluid equation is that there is a density and pressure buildup just below the potential drop. In the fluid formalism the maximum density was explicitly given by $\hat{n_{0}}$. If there were no density enhancement by the potential drop, we would not get a linear CV-relationship, but rather a square root $\mathrm{CV}$-relationship, as can be easily seen from our formulas. A simple physical explanation is that if the density were constant, the current would be given by $e n v_{f}$ where $v_{f}$ is the final velocity of the electrons accelerated by the potential drop $V$. Since $v_{f} \propto \sqrt{V}$ (assuming a nonrelativistic case), the current density would also be proportional to $\sqrt{V}$. However, we have thus far completely neglected the ions in both kinetic and fluid theory. Thus, if there is an electron density enhancement below the potential drop, there must also be an ion density enhancement, in order to maintain quasineutrality. To study this question further we need a theory which includes the ions and where the parallel electric field profile is determined selfconsistently. One possibility is to use the one-fluid formalism, where the electron and ion momentum equations are "summed and subtracted" to give the center of mass momentum equations and generalized Ohm's law. This will be the subject of the next section.

A problem of gas escape through a hole in the container wall has been treated in both fluid and kinetic theories (Gombosi, 1994), with the remark that kinetic theory applies to this problem when the hole diameter is much smaller than the mean free path whereas fluid theory applies in the reverse limit. The formulas obtained are the same as ours and those given by (Landau and Lifshitz (1987), despite the fact that the geometry is different. Applying this line of thought, in our case kinetic theory should be a better approximation than fluid theory because the mean free path is very large compared to the system size.

\subsection{Conclusion}

The electron fluid model reproduces, apart from a numerical factor, the kinetic theory CV-relationship if and only if $\gamma=3$, corresponding to motion with only one degree of freedom. For this to happen it is essential that the density below the potential drop is enhanced, otherwise a square root $\mathrm{CV}$-relationship would result for any value of $\gamma$.

\section{One-fluid theory}

\subsection{Derivation of basic equations}

The one-fluid equations are given by (Seyler, 1990)

$$
\begin{aligned}
& \frac{\partial n}{\partial t}+\nabla \cdot(n \mathbf{v})=0 \\
& m_{i} n \frac{\mathrm{d} \mathbf{v}}{\mathrm{d} t}=\mathbf{j} \times \mathbf{B}-\nabla P^{\mathrm{CM}} \\
& \frac{\partial \mathbf{B}}{\partial t}=-\nabla \times \mathbf{E} \\
& \nabla \times \mathbf{B}=\mu_{0} \mathbf{j} \\
& \mathbf{E}+\mathbf{v} \times \mathbf{B} \\
& =\frac{m_{e}}{n e^{2}}\left[\frac{\partial \mathbf{j}}{\partial t}+\nabla \cdot(\mathbf{v j}+\mathbf{j} \mathbf{v})\right]+\frac{1}{e n} \mathbf{j} \times \mathbf{B}-\frac{1}{e n} \nabla P_{e}^{\mathrm{CM}} \\
& \frac{\mathrm{d}}{\mathrm{d} t}\left(P^{\mathrm{CM}} n^{-\gamma}\right)=0 .
\end{aligned}
$$

The symbol $P_{e}^{\mathrm{CM}}$ denotes the electron pressure computed with respect to the center of mass velocity $\mathbf{v} \equiv\left(m_{e} \mathbf{v}_{e}+m_{i} \mathbf{v}_{i}\right) /\left(m_{e}+m_{i}\right)$. Likewise, $P^{\mathrm{CM}}$ is the total (electon plus ion) center-of-mass pressure. The connection between center-of-mass and ordinary pressure gradients are (Krall and Trivelpiece, 1973)

$$
\begin{aligned}
& \nabla P_{e}^{\mathrm{CM}}=\nabla P_{e}+\frac{m_{e}}{e^{2}} \mathbf{j} \cdot \nabla\left(\frac{\mathbf{j}}{n}\right) \\
& \nabla P_{i}^{\mathrm{CM}}=\nabla P_{i}+\frac{m_{e}^{2}}{m_{i} e^{2}} \mathbf{j} \cdot \nabla\left(\frac{\mathbf{j}}{n}\right) .
\end{aligned}
$$

Now we assume a stationary state $(\partial / \partial t=0)$, a strong external magnetic field (low-beta plasma), quantities depending only on the field-aligned coordinate denoted by $s$, and vectors $\mathbf{v}$ and $\mathbf{j}$ having only field-aligned components denoted by $v_{\|}, j_{\|}$(positive downward). Let us again denote $\boldsymbol{\Pi} \equiv n \mathbf{v}$. Under these assumptions we obtain

$$
\begin{aligned}
& P^{\mathrm{CM}}=C n^{\gamma} \\
& \mathbf{\Pi}=\Pi_{0}\left(\frac{b}{b_{0}}\right) \\
& j_{\|}=j_{0}\left(\frac{b}{b_{0}}\right) \\
& m_{i} n[\mathbf{v} \cdot \nabla \mathbf{v}]_{\|}=-\frac{\mathrm{d} P^{\mathrm{CM}}}{\mathrm{d} s} \\
& E_{\|}=\frac{m_{e}}{n e^{2}}\left[\nabla \cdot(\mathbf{v} \mathbf{j}+\mathbf{j} \mathbf{v})-\mathbf{j} \cdot \nabla\left(\frac{\mathbf{j}}{e n}\right)\right]_{\|}-\frac{1}{e n} \frac{\mathrm{d} P_{e}}{\mathrm{~d} s} .
\end{aligned}
$$


where $\Pi_{0}, j_{0}$ and $b_{0}$ are the ionospheric mass flux, current density and normalized magnetic field, respectively (the other quantities with zero subscript such as $n_{0}, P_{0}, T_{0}$ are magnetospheric variables).

With the help of lemma (45) we compute (identifying $\mathbf{U}=\mathbf{V}=n \mathbf{v})$

$$
\begin{aligned}
{[\mathbf{v} \cdot \nabla \mathbf{v}]_{\|} } & =\left[\frac{1}{n}(n \mathbf{v}) \cdot \nabla\left(\frac{1}{n} n \mathbf{v}\right)\right]_{\|} \\
& =\left[\frac{1}{n^{2}}(n \mathbf{v}) \cdot \nabla(n \mathbf{v})-\frac{1}{n} \mathbf{v} \cdot \nabla n\right]_{\|} \\
& =v_{\|}^{2}\left(\frac{1}{b} \frac{\mathrm{d} b}{\mathrm{~d} s}-\frac{1}{n} \frac{\mathrm{d} n}{\mathrm{~d} s}\right)
\end{aligned}
$$

Let us now compute $[\nabla \cdot(\mathbf{v j}+\mathbf{j} \mathbf{v})]_{\|}$:

$$
\begin{aligned}
& {[\nabla \cdot(\mathbf{v j}+\mathbf{j} \mathbf{v})]_{\|}=[(\nabla \cdot \mathbf{v}) \mathbf{j}+\mathbf{v} \cdot \nabla \mathbf{j}+\mathbf{j} \cdot \nabla \mathbf{v}]_{\|}} \\
& {[(\nabla \cdot \mathbf{v}) \mathbf{j}]_{\|}=-\frac{1}{n} j_{\|} v_{\|} \frac{\mathrm{d} n}{\mathrm{~d} s}} \\
& {[\mathbf{v} \cdot \nabla \mathbf{j}]_{\|}=\frac{1}{n}[n \mathbf{v} \cdot \nabla \mathbf{j}]_{\|}=v_{\|} j_{\|} \frac{1}{b} \frac{\mathrm{d} b}{\mathrm{~d} s}} \\
& {[\mathbf{j} \cdot \nabla \mathbf{v}]_{\|}=\left[\mathbf{j} \cdot \nabla\left(\frac{1}{n} n \mathbf{v}\right)\right]_{\|}} \\
& =j_{\|} v_{\|}\left(\frac{1}{b} \frac{\mathrm{d} b}{\mathrm{~d} s}-\frac{1}{n} \frac{\mathrm{d} n}{\mathrm{~d} s}\right),
\end{aligned}
$$

thus

$[\nabla \cdot(\mathbf{v j}+\mathbf{j} \mathbf{v})]_{\|}=2 j_{\|} v_{\|}\left(\frac{1}{b} \frac{\mathrm{d} b}{\mathrm{~d} s}-\frac{1}{n} \frac{\mathrm{d} n}{\mathrm{~d} s}\right)$.

We also need to compute $\mathbf{j} \cdot \nabla(\mathbf{j} / n)$ :

$$
\left[\mathbf{j} \cdot \nabla\left(\frac{\mathbf{j}}{n}\right)\right]_{\|}=\frac{j_{\|}^{2}}{n}\left(\frac{1}{b} \frac{\mathrm{d} b}{\mathrm{~d} s}-\frac{1}{n} \frac{\mathrm{d} n}{\mathrm{~d} s}\right) .
$$

Switching to variable $b$, the momentum equation [Eq. (68)] and Ohm's law (69) now assume the form

$$
\begin{gathered}
m_{i} \frac{\Pi_{0}^{2}}{n}\left(\frac{b}{b_{0}}\right)^{2}\left(\frac{1}{b}-\frac{1}{n} \frac{\mathrm{d} n}{\mathrm{~d} b}\right)=-\frac{\mathrm{d} P^{\mathrm{CM}}}{\mathrm{d} b} \\
E_{\|}=\left[\frac{m_{e}}{n^{2} e^{2}} j_{0}\left(\frac{b^{2}}{b_{0}^{2}}\right)\left(\frac{1}{b}-\frac{1}{n} \frac{\mathrm{d} n}{\mathrm{~d} b}\right)\right. \\
\left.\times\left(2 \Pi_{0}-\frac{j_{0}}{e}\right)-\frac{1}{e n} \frac{\mathrm{d} P_{e}}{\mathrm{~d} b}\right] \frac{\mathrm{d} b}{\mathrm{~d} s} .
\end{gathered}
$$

This pair of fundamental equations determines the profiles of $n$ and $E_{\|}$. The plasma flux and the current flowing through the flux tube are parametrized by $\Pi_{0}$ and $j_{0}$, respectively. The situation is different than in the previous section where we considered only the electron fluid. In that case $E_{\|}$was not self-consistently determined, but in one-fluid theory it is.

\subsection{Solution}

Let us first consider the momentum equation [Eq. (77)]. After substituting $P^{\mathrm{CM}}=C n^{\gamma}$ it can be written in the form

$$
\left[C \gamma n^{\gamma-2}-m_{i} \Pi_{0}^{2}\left(\frac{b}{b_{0}}\right)^{2} \frac{1}{n^{3}}\right] \frac{\mathrm{d} n}{\mathrm{~d} b}+m_{i}\left(\frac{\Pi_{0}}{b_{0}}\right)^{2} \frac{b}{n^{2}}=0 .
$$

This differential equation is exact (Spiegel, 1968) and thus it can be integrated easily. Unfortunately the solution can not be analytically expressed as $n=n(b)$ but only in the inverse form, $b=b(n)$. After using the source plasma boundary condition $n(b=1)=n_{0}$ we obtain

$$
b(n)=\frac{n b_{0}}{\Pi_{0}}\left[\left(\frac{\Pi_{0}}{n_{0} b_{0}}\right)^{2}+\frac{2 C}{m_{i}}\left(\frac{\gamma}{\gamma-1}\right)\left(n_{0}^{\gamma-1}-n^{\gamma-1}\right)\right]^{1 / 2},
$$

where $C=T_{0} / n_{0}^{\gamma-1}$.

The behavior of $b(n)$ is shown in Fig. 10. For large enough $\Pi_{0}, b(n)$ has a maximum as a function of $n$ in the domain $1 \leq b \leq b_{0}$. The maximum occurs where $\mathrm{d} b / \mathrm{d} n=0$ and at the maximum

$$
\begin{aligned}
n & =n_{\max } \\
& =n_{0}\left[\frac{2}{\gamma+1}+\frac{\gamma-1}{\gamma(\gamma+1)} \frac{1}{b_{0}^{2}} \frac{m_{i}}{T_{0}}\left(\frac{\Pi_{0}}{b_{0} n_{0}}\right)^{2}\right]^{\frac{1}{\gamma-1}} .
\end{aligned}
$$

If we require that the maximum as a function of $n$ occurs exactly at $b=b_{0}$ (that is, $\mathrm{d} b / \mathrm{d} n=0$ at $b=b_{0}$ ), we obtain a condition for the mass flux $\Pi_{0}$ :

$$
\begin{aligned}
\Pi_{0} & =n_{0} \sqrt{\frac{\gamma T_{0}}{m_{i}}} \\
& {\left[\frac{2}{\gamma+1}+\frac{\gamma-1}{\gamma(\gamma+1)} \frac{1}{b_{0}^{2}} \frac{m_{i}}{T_{0}}\left(\frac{\Pi_{0}}{b_{0} n_{0}}\right)^{2}\right]^{\frac{1}{2}+\frac{1}{\gamma-1}} . }
\end{aligned}
$$

For large $b_{0}$, which is usually a good approximation, the last term can be dropped and we obtain

$$
\Pi_{0}=n_{0} \sqrt{\frac{\gamma T_{0}}{m_{i}}}\left(\frac{2}{\gamma+1}\right)^{\frac{\gamma+1}{2(\gamma-1)}} \text {. }
$$

This is exactly the same relation as Eq. (51). That is, the requirement that $b(n)$ is marginally single-valued leads to the same condition as marginally choked flow at the

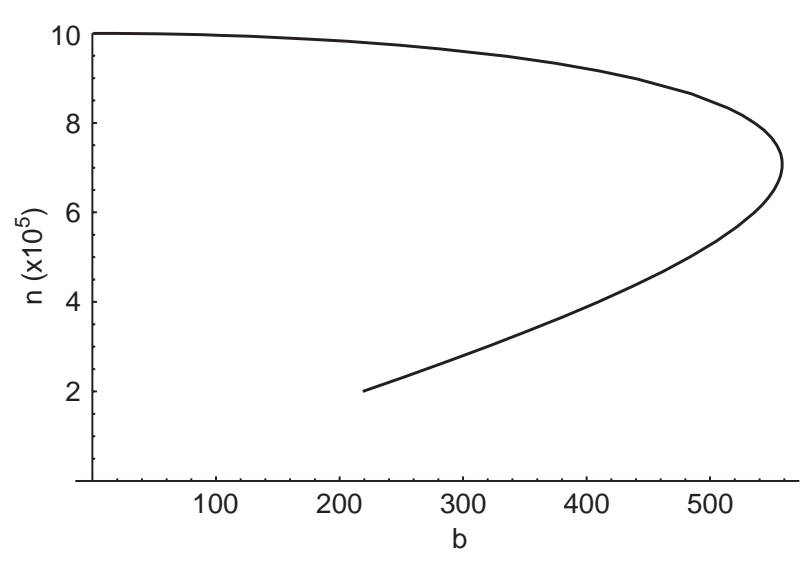

Fig. 10. Behavior of Eq. (80) 
"nozzle exit" (the ionosphere). Notice that in the onefluid equations the electric field does not appear in the momentum equation at all because of quasineutrality and thus the momentum equation could be solved first, independently of Ohm's law.

Using Eqs. (78) and (80), the computation of the potential $V=-\int_{0}^{s} d s E_{\|}$can be done analytically. Omitting the details of the calculation, the result is

$$
\begin{aligned}
V(n)= & \frac{m_{e}}{m_{i}}\left(\frac{j_{0}}{e \Pi_{0}}\right)^{2} \frac{T_{0}}{e}\left(\frac{\gamma}{\gamma-1}\right)\left(1-\frac{2 \Pi_{0} e}{j_{0}}\right) \\
& \times\left[1-\left(\frac{n}{n_{0}}\right)^{\gamma-1}\right]-\frac{T_{0 e}}{e} \frac{\gamma_{e}}{\gamma_{e}-1}\left[1-\left(\frac{n}{n_{0}}\right)^{\gamma_{e}-1}\right] .
\end{aligned}
$$

This gives the potential drop as a function of the density. To produce the potential drop as a function of $b$, the relationship (80) must be numerically inverted and substituted in Eq. (84). Doing this, we find that for sufficiently negative (i.e., upward) $j_{0}$, the potential $V$ becomes positive (ionosphere at higher potential than magnetosphere). In Fig. 11 we plot a typical $V(b)$ behavior.

From Fig. 11 we see that the potential drop is concentrated at a low altitude, which is at odds with observations and also badly violates the FL-condition (7) of the kinetic theory. To study this discrepancy further, we note that it is also possible to see the rough meaning of Eq. (84) analytically, without doing the numerical inversion. First we note that usually the ion flux is much smaller than the electron flux, $\Pi_{0} \ll j_{0} / e$. Likewise, reasonable solutions of Eq. (80) are such that the ionospheric density $n\left(b_{0}\right)$ is some fraction of the source density $n_{0}$, and at the same time, $\Pi_{0}$ is proportional to $n_{0} \sqrt{T_{0} / m_{i}}$. Putting these together we see that Eq. (84) yields approximately

$V\left(n\left(b_{0}\right)\right) \equiv \Delta V=A_{1} \frac{m_{e} j_{0}^{2}}{e^{3} n_{0}^{2}}-A_{2} \frac{T_{0 e}}{e}$

where $A_{1}, A_{2}$ are numerical factors of order unity. Solving for $j_{0}$ thus yields a square root $\mathrm{CV}$-relationship, as for the electron fluid theory with constant density.

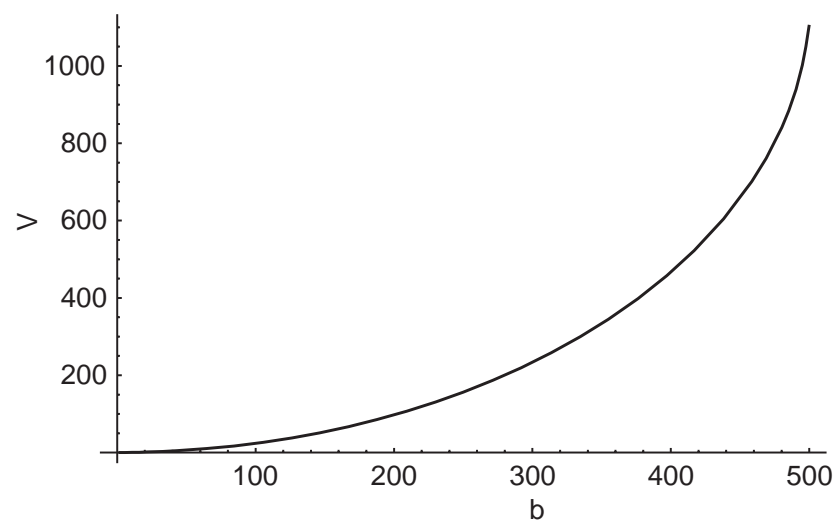

Fig. 11. When $n=n(b)$ is inverted form Eq. (80) and the result is substituted into Eq. (84), the result is this $V=V(b)$ for sufficiently negative $j_{0}$
The square root dependency is such that for $V \propto T_{e} / e$, numerically almost equivalent results are obtained as for the kinetic theory. ("Almost" means up to a numerical factor of order unity.)

\subsection{Conclusion}

Inclusion of magnetospheric ion dynamics by the onefluid model suppresses the electron density enhancement below the potential drop and thus produces a square root CV-relationship. At the same time, the potential drop is concentrated at a low altitude, which is physically unreasonable. This means that the one-fluid theory composed of magnetospheric electrons and protons is unable to produce realistic results. It cannot answer the question of what could maintain a realistic potential drop in a self-consistent way. The physical reason for the failure of the one-fluid theory is very simple: the ions are pushed upward, not downward, by an upward electric field, and thus the magnetospheric ions are unable to provide quasineutrality for any electron density enhancement below the potential drop.

\section{Four-fluid theory}

In this section we consider the ionosphere as a plasma source, not only as an absorber of magnetospheric particles. The idea is to study whether the ionospheric ions can neutralize the charge cloud of the electron density enhancement below the potential drop and thus resolve the puzzle that we ended up with in the previous section.

We shall treat the magnetospheric and ionospheric electrons and ions as four separate fluids moving in an electrostatic potential. The potential should be determined from the quasineutrality requirement.

Using a derivation similar to what was performed in the start of section "Electron fluid theory" we derive, for each fluid, equations similar to Eq. (48). Let us denote the charge of the fluid by $q$, ionospheric variables $\left(b=b_{0}\right)$ by subscript $i$ and magnetospheric variables $(b=1)$ by subscript $m$. For fluids which have an ionospheric boundary condition $n\left(b_{0}\right)=n_{i}$ we obtain

$$
\begin{aligned}
& C \frac{\gamma}{\gamma-1}\left(n^{\gamma-1}-n_{i}^{\gamma-1}\right)+\frac{1}{2} m \Pi_{0}^{2}\left(\frac{b^{2}}{b_{0}^{2} n^{2}}-\frac{1}{n_{i}^{2}}\right) \\
& \quad=q\left(V\left(b_{0}\right)-V(b)\right)
\end{aligned}
$$

where $C=T_{i} / n_{i}^{\gamma-1}$. For fluids of magnetospheric origin we obtain $\left(n(1)=n_{m}\right)$

$$
\begin{aligned}
& C \frac{\gamma}{\gamma-1}\left(n^{\gamma-1}-n_{m}^{\gamma-1}\right)+\frac{1}{2} m \Pi_{0}^{2}\left(\frac{b^{2}}{b_{0}^{2} n^{2}}-\frac{1}{b_{0}^{2} n_{m}^{2}}\right) \\
& \quad=q(V(1)-V(b))
\end{aligned}
$$

where $C=T_{m} / n_{m}^{\gamma-1} . \Pi_{0}$ is the mass flux at the ionospheric plane in each case and $m$ is the particle mass. We will normalize the potential $V$ so that $V(1)=0$, that is, the potential is zero deep in the magnetosphere. Positive direction is towards the ionosphere. 
We can write both Eqs. (86) and (87) in the form

$n^{\gamma-1}+\frac{\alpha}{n^{2}}=\beta$

where

$\alpha=\frac{1}{2} m \Pi_{0}^{2}\left(\frac{b}{b_{0}}\right)^{2}\left(\frac{\gamma-1}{\gamma}\right) \frac{1}{C}$

for both ionospheric and magnetospheric fluids and

$\beta=n_{i}^{\gamma-1}+\left(\frac{b_{0}}{b}\right)^{2} \frac{\alpha}{n_{i}^{2}}+\left(\frac{\gamma-1}{\gamma}\right) \frac{1}{C} q\left(V\left(b_{0}\right)-V(b)\right)$

for the ionospheric case, and

$\beta=n_{m}^{\gamma-1}+\left(\frac{1}{b}\right)^{2} \frac{\alpha}{n_{m}^{2}}+\left(\frac{\gamma-1}{\gamma}\right) \frac{1}{C} q(-V(b))$

for the magnetospheric case.

In Fig. 12 we show the behavior of Eq. (88). This nonlinear equation has in general two roots. The minimum of the curve $n^{(\gamma-1)}+\alpha / n^{2}$ is reached at the zero of the derivative, which is given by

$n_{\min }=\left(\frac{2 \alpha}{\gamma-1}\right)^{1 /(\gamma+1)}$.

If $\beta<n_{\min }^{\gamma-1}+\alpha / n_{\min }^{2}$, there are no roots. Otherwise there are two roots (or one root in case of exact equality) for positive $n$, one of which is smaller than $n_{\min }$ and the other one larger than $n_{\min }$.

\subsection{Ionospheric electrons and ions}

Magnetospheric electrons and protons will follow equations similar to those presented in section "Electron fluid theory", but the ionospheric particles need rather different treatment. Let us first consider ionospheric electrons. We require that, at the ionosphere, the two roots of Eq. (88) coincide and are equal to some prescribed ionospheric source density. This provides the largest possible escape of ionospheric particles that is possible within the framework of fluid theory. From Eqs. (89) and (90) we can see that this happens if $n_{i}=n_{\min }$ at $b=b_{0}$. Using Eq. (92) this implies

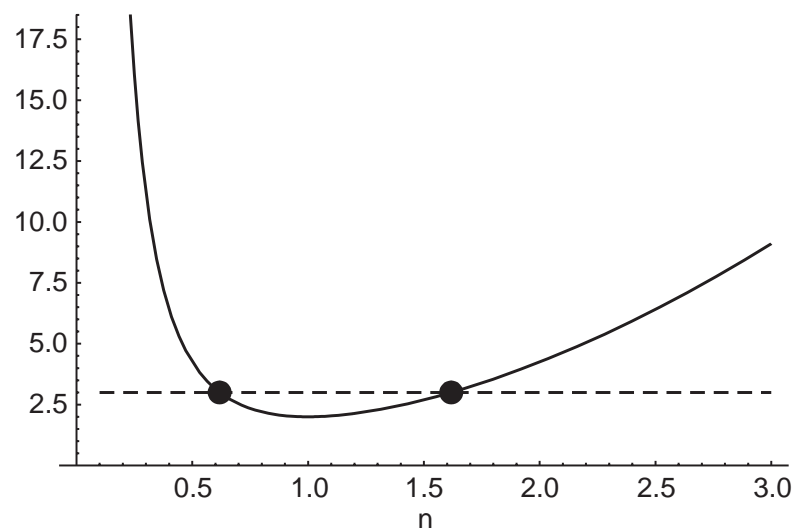

Fig. 12. The curve $n^{\gamma-1}+\alpha / n^{2}$ as a function of $n$ has, in general, two roots
$\Pi_{0}=n_{i} \sqrt{\frac{\gamma T_{i}}{m}}$.

Actually, the square root expression in Eq. (93) is equal to the local sound speed, thus the requirement that the two roots coincide at the ionosphere leads to a prediction of transonic escape flux. The lower of the two roots (i.e., the one corresponding to smaller density) corresponds to a supersonic escape. In this solution, the density decreases upward, at least if $V=0$. In the other branch the density would increase upward, and this would correspond to subsonic flow. The subsonic branch is nonphysical.

In Fig. 13 we have plotted the supersonic solution for ionospheric electrons varying the potential drop. We used negative potential drops of the order of a few volts. When the potential drop is made more negative, the density drops at a fixed height, which is a reflection of the fact that the electron upward velocity increases as the electric field accelerates the electrons upward. Notice, however, that in our model the escape flux does not depend on the potential drop, because the ionosphere is considered an infinite half-space reservoir where the electron density is assumed to be fixed by some (unspecified) ionospheric processes. In reality, a negative potential drop of the order of a few volts or more would most probably increase the escape flux and try to empty the ionosphere of electrons, resulting in a neutralization of the potential drop (Fridman and Lemaire, 1980).

If the potential drop is positive (corresponding to electron precipitation), the two roots start to coincide also in the magnetosphere for finite $b$, quickly producing a situation where we have no solution in most or all of the domain. This happens already for quite small potentials, a fraction of a volt. The physical reason is clear: we are trying to emit electrons from the ionosphere by Eq. (93), which is impossible if the electric

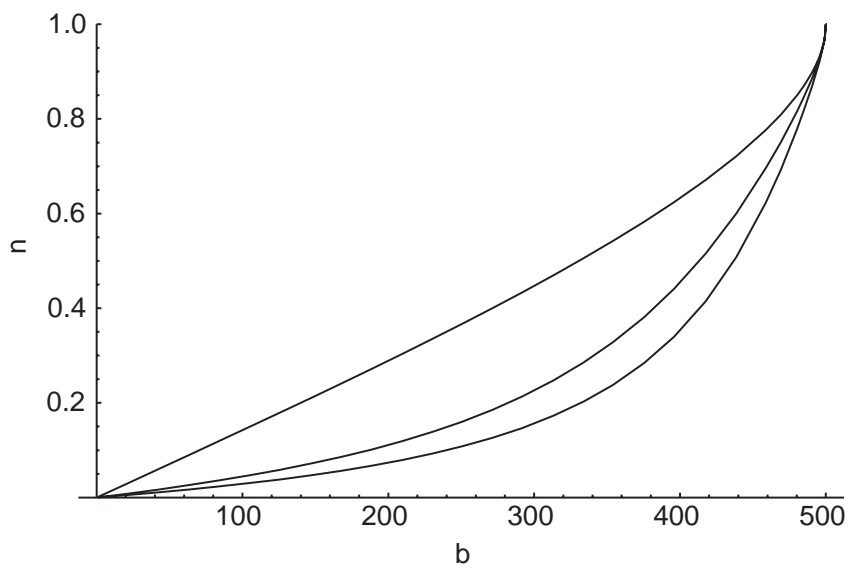

Fig. 13. Ionospheric electron escape solutions (smaller of the two roots chosen) for (88) for zero, $-2 \mathrm{~V}$ and $-5 \mathrm{~V}$ potential drops from top to bottom, respectively. Negative potential drop means that the ionosphere is in a lower potential than the magnetosphere, thus the parallel electric field tries to pull electrons from the ionosphere. The electron density at a fixed height $(b=200$, say) decreases when the potential drop becomes more negative because electrons move faster and the flux must stay constant 
field is upward. The right thing to do is to shut off the ionospheric electron emission in this case. The details of how this actually happens are outside the scope of our equations, but in this study we are interested in potential drops very much larger than the ionospheric electron temperature and for these potential drops simply removing ionospheric electrons altogether from the model is perfectly adequate.

For ionospheric ions the situation is completely similar as for ionospheric electrons, only the sign of the potential drop must be reversed to get an equivalent situation. That is, the emission of ionospheric ions must be shut off if the potential drop is negative (a noninteresting situation in this work). For positive potential drops, curves similar to Fig. 13 are obtained. For any substantial positive potential drops (more than $100 \mathrm{~V}$, say) the approximation $q V \gg T_{i}$ is perfectly valid and the term $n^{\gamma-1}$ in Eq. (88) can be neglected everywhere but in the immediate vicinity of the ionosphere. Under this approximation the solution for $n$ is given by $n \approx \sqrt{\alpha / \beta}$. This can be written also as

$n(b) \approx\left(\frac{b}{b_{0}}\right) n_{i} \sqrt{\frac{\gamma T_{i}}{2 q\left(V\left(b_{0}\right)-V(b)\right)}}$,

from which we see that $n / b$ is inversely proportional to the square root of the potential. This is understandable since the potential difference increases the kinetic energy of the particles linearly. (We have $n / b$ rather than $n$ because of the flux tube scaling.)

We are interested in an upward electric field case (positive potential drop), which is significantly larger than the ionospheric temperatures. Thus we can neglect ionospheric electrons, and use Eq. (94) above the ionosphere for ionospheric ions. Thus we do not have to solve any nonlinear equation to get the response of the ionospheric particles to an applied positive potential drop. We would like to investigate whether or not Eq. (94) can neutralize the charge cloud of the electron density bump of magnetospheric electrons. Looking at Eq. (94), it is clear that for this to succeed, the density bump must occur in a region where the potential is nearly the same as the ionospheric potential, that is, $V\left(b_{0}\right)-V(b)$ must be small (of the same order as $T_{i} / q$ ). Otherwise the ionospheric ions will move fast upward and their density will be low because of the continuity equation.

\subsection{A self-consistent solution sketched}

The quasi-neutrality constraint means that $n_{e}=n_{i}+n_{p}$, where $e, i, p$ refer to electrons, ionospheric ions, and protons, respectively. Electrons and protons are of magnetospheric origin. We know how to compute each of these densities once the potential $V(b)$ is specified. Finding a self-consistent $V(b)$ which satisfies quasineutrality is thus a matter of solving a nonlinear equation for each $b$, Eq. (88). Doing this analytically seems to be impossible, and doing it numerically is difficult because it requires solving a strongly nonlinear equation, which has potentially many solutions. Furthermore, to compute each of the densities (for example, $n_{p}$ ) itself requires numerical root finding, so we would end up with two nested nonlinear equations, except perhaps for $n_{i}$, for which the approximate Eq. (94) could be used. To get some insight into the problem, we want to develop a simplified analytically tractable model.

Consider a situation where the potential drop (corresponding to upward electric field) is relatively well localized in the acceleration region, which resides at high enough altitude such that $b \ll b_{0}$ at the potential drop, (see again Fig. 8). Then we can neglect the flow of magnetospheric populations within the acceleration region, and thus relate the fluid states below and above the potential drop by equations similar to Eq. (52). Below the potential drop we compute the density profiles by assuming that the escape into ionosphere is "choked", which is described by Eq. (51). We do this for magnetospheric electrons and protons independently.

Above the acceleration region the magnetospheric particles maintain quasineutrality by themselves (the densities are uniform and equal to the source plasma density). Below the acceleration region the electron density $n_{e}$ is higher than the proton density $n_{p}$, and their difference profile depends on the strength of the potential drop. The difference must be made equal to the density of ionospheric ions, $n_{i}=n_{e}-n_{p}$. Thus we must construct a model for ionospheric ion outflow such that $n_{i}$ is a given function of height below the acceleration region. Since $n_{i}(b)$ follows formula (94) to a good approximation, we see that this is in fact easy to accomplish just by solving $V(b)$ from Eq. (94). The $V(b)$ variations obtained will be of the order of ionospheric energies and thus much smaller than the acceleration region potential drop. Thus these variations do not affect the motion of the hot magnetospheric populations and thus their motion below the acceleration region can be solved by assuming zero electric field, i.e., the normal fluid-dynamical choked flow. The electric field will point upward everywhere and thus quench ionospheric electron outflow. Thus ionospheric electrons can be forgotten; all electrons are hot and of magnetospheric origin. Thus our fourfluid model is actually a three-fluid model.

Nevertheless, some ionospheric electrons could, in reality, reach the acceleration region by their thermal velocity. However, they cannot move above it, thus the net upward flow of ionospheric electrons must be zero in any case. If ionospheric electrons do exist below the acceleration region, then the solution for $V(b)$ below the acceleration region is slightly modified, but this does not affect the magnetospheric precipitating populations. What we have shown in this subsection is that it is possible to get one solution without ionospheric electrons, and we think that this is the most reasonable solution because the electric field points upward anyway.

Above the acceleration region the density $n_{i}$ should be small in order not to break the already existing quasineutrality $\left(n_{e}=n_{p}\right)$. This is automatically satisfied. As the potential drop is much larger than ionospheric energies, the density $n_{i}$ above the acceleration region will 
be orders of magnitude smaller than below (and the upward ion velocity will be correspondingly larger).

The CV-relationship from this model will exactly match the results obtained in section "Electron fluid theory" (see especially Eq. 56). The electron fluid will behave exactly similarly to that was described in that previous section. What we have added here is an explanation how self-consistency can be preserved by the addition of ionospheric ion outflow. Thus a linear $\mathrm{CV}$-relationship is obtained if $\gamma=3$ for magnetospheric electrons.

In this simple model we take no stand on the internal structure of the acceleration region. Nor do we try to explain why the acceleration region resides at a certain altitude. Probably the acceleration region altitude somehow depends on the ionospheric ion flux. These questions, as well as finding a quantitative realization of the model sketched here, are left for a future study. It would also be of importance to study whether these ideas could be translated into the language of one-fluid theory. The plasma emitted by the ionosphere in this case consists only of ions and thus is not quasineutral; thus one probably has to do more than just add a suitable ionospheric boundary condition to the one-fluid equations written here. Quasineutrality is achieved only when ionospheric and magnetospheric plasmas overlap the same region.

\subsection{Conclusion}

A linear CV-relationship can emerge again if we take into account outflowing ionospheric ions. All the conclusions of section "Electron fluid theory" remain valid.

\section{Summary}

Our principal long-term aim is to develop simulations of smaller scale auroral phenomena such as individual arcs. Even though the scale sizes may be small in the perpendicular direction, usually the whole field line length from the ionosphere to the equatorial plane should be included in the simulation box in order to achieve realism. This requirement, by and large, prohibits us from using self-consistent electrostatic particle simulations, because the simulation box would contain too many Debye spheres. Thus fluid simulations are the only computationally feasible alternative. On the other hand, the current-voltage (CV) relationship is an essential observational fact. It can be derived from kinetic theory. Here we explored possibilities to make these two requirements meet: how to build a fluid simulation which reproduces the correct CV-relationship. We summarize our findings:

1. We have computed the density and pressure profiles analytically from electron kinetic theory. These formulas reveal that the pressure and density are enhanced below the potential drop, and decrease again near the ionosphere.
2. Obtaining a linear $\mathrm{CV}$-relationship requires that the electron density is enhanced below the potential drop. If the density stays constant, a square root CVrelationship results. This is so because the final velocity of accelerated electrons is proportional to the square root of the accelerating voltage (neglecting electron temperature).

3. The linear CV-relationship is reproduced in electron fluid theory only if $\gamma=3$. The numerical constants differ somewhat from kinetic theory even for $\gamma=3$, however.

4. One-fluid theory, while aiming at self-consistency, cannot reproduce a linear CV-relationship. Instead, it predicts a square root CV-relationship. The parallel potential drop is concentrated on a low altitude, just above the ionosphere, which is unrealistic.

5. The relevant terms in generalized Ohm's law are the electron pressure term and electron inertial terms.

6. It is very important in this application to distinguish between the center-of-mass electron pressure $P_{e}^{\mathrm{CM}}$ and the 'normal' electron pressure $P_{e}$. If this distinction is not properly made, completely wrong results are obtained.

7. Inclusion of upwelling ionospheric ions is able to restore the electron fluid theory results in a selfconsistent manner. The basic idea is that below acceleration region a charge imbalance between magnetospheric electrons and protons will develop, which is balanced by ionospheric ions. Above acceleration region the ionospheric ions move upward so fast that their density is very small, thus they do not affect the charge balance there.

Acknowledgements. The authors thanks Kirsti Kauristie and Annika Olsson for reading the manuscript and making a number of useful corrections and suggestions.

Topical Editor K.-H. Glassmeier thanks R. Lysak and H. Voigt for their help in evaluating this paper.

\section{References}

Abramowitz, M., and I. A. Stegun, Handbook of mathematical functions, Dover, New York, 1972.

Anderson, J.D., Jr., Modern compressible flow with historical perspective, 2nd edn, McGraw-Hill, 1990.

Fridman, M., and J. Lemaire, Relationship between auroral electron fluxes and field aligned electric potential differences, J. Geophys. Res., 85, 664-670, 1980.

Chiu, Y. T., and M. Schulz, Self-consistent particle and parallel electrostatic field distributions in the magnetospheric-ionospheric auroral region, J. Geophys. Res., 83, 629-642, 1978.

Gombosi, T. I., Gaskinetic theory, Cambridge University Press, Cambridge, 1994.

Haerendel, G., H. U. Frey, O. H. Bauer, E. Rieger, J. Clemmons, M. H. Boehm, D. D. Wallis, and H. Lühr, Inverted-V events simultaneously observed with the Freja satellite and from the ground, Geophys. Res. Lett., 21, 1891-1894, 1994.

Janhunen, P., GUMICS-3 - a global ionosphere magnetosphere coupling simulation with high ionospheric resolution, in Proc. Environmental Modelling for Space-Based Applications. 18-20 Sept. 1996, ESTEC, The Netherlands, 1996.

Janhunen, P., and A. Olsson, The current-voltage relationship revisited: Exact and approximate formulas with almost general 
validity for hot magnetospheric electrons for bi-Maxwellian and kappa distributions. Ann. Geophysicae., 16, 292-297, 1998.

Janhunen, P., and Koskinen, H. E. J., The closure of Region-1 fieldaligned current in MHD simulation, Geophys. Res. Lett., 24, 1419-1422, 1997.

Knight, S., Parallel electric fields, Planet. Space Sci., 21, 741-750, 1973.

Krall, N. A., and A. W. Trivelpiece, Principles of plasma physics, McGraw-Hill, New York, 1973.

Landau, L. D., and E. M. Lifshitz, Fluid mechanics, 2nd edn, Course of theoretical physics, vol 6, Pergamon Pres, New York, 1987.

Lu, G., P. H. Reiff, J. L. Burch, and J. D. Winningham, On the auroral current-voltage relationship, J. Geophys. Res., 96, 3523$3531,1991$.

Lundin, R., Investigations of auroral electron precipitation by means of sounding rockets, KGI REPORT NO. 77:1 April 1977.

Lundin, R., and I. Sandahl, Some characteristics of the parallel electric field acceleration of electrons over discrete auroral arcs as observed from two rocket flights, ESA SP-135, 125-136, 1978.

Lyons, L. R., D. S. Evans, and R. Lundin, An observed relation between magnetic field aligned electric fields and downward electron energy fluxes in the vicinity of auroral forms, $J$. Geophys. Res., 84, 457-461, 1979.
Olsson, A., Studies of magnetosphere-ionosphere coupling in auroral substorms using radar and satellite techniques, $\mathrm{Ph} \mathrm{D}$ thesis, IRF Scientific Report 240, Kiruna, 1997.

Olsson, A., A. I. Eriksson, and P. Janhunen, On the current-voltage relationship in auroral breakups and westward-traveling surges, Ann. Geophysicae, 14, 1265-1273, 1996.

Olsson, A., L. Andersson, A. I. Eriksson, J. Clemmons, R. E. Erlandson, G. Reeves, T. Hughes, and J. S. Murphree, Freja studies of the current-voltage relation in substorm related events, J. Geophys. Res., 103, 4285-4301, 1998.

Pierrard, V., New model of magnetospheric current-voltage relationship, J. Geophys. Res., 101, 2669-2675, 1996.

Sakanoi, K., H. Fukunishi, and T. Mukai, Relationship between fieldaligned currents and inverted-V parallel potential drops observed at midaltitudes, J. Geophys. Res., 100, 19 343-19 360, 1995.

Seyler, C. E., A mathematical model of the structure and evolution of small-scale discrete auroral arcs, J. Geophys. Res., 95, 17 199-17 215, 1990.

Spiegel, M. R., Mathematical handbook of formulas and tables, Schaum's Outline Series in Mathematics, McGraw-Hill, 1968.

Weimer, D. R., D. A. Gurnett, C. K. Goertz, J. D. Menietti, J. L. Burch, and M. Sugiura, The current-voltage relationship in auroral current sheets, J. Geophys. Res., 92, 187-194, 1987.

Whipple, E. C., The signature of parallel electric fields in a collisionless plasma, J. Geophys. Res., 82, 1525-1531, 1978. 